To appear in Water Resources Res. 39(0),XXXX, doi:10.1029/2002WR001802, 2003

\title{
A Stochastic Model of Space-Time Variability of Mesoscale Rainfall: Statistics of Spatial Averages
}

\author{
Prasun K. Kundu \\ Goddard Earth Sciences and Technology Center, University of Maryland Baltimore County, \\ Baltimore, MD and NASA/Goddard Space Flight Center, Greenbelt, MD
}

Thomas L. Bell

Laboratory for Atmospheres, NASA/Goddard Space Flight Center, Greenbelt, MD

\begin{abstract}
A characteristic feature of rainfall statistics is that they depend on the space and time scales over which rain data are averaged. A previously developed spectral model of rain statistics that is designed to capture this property, predicts power law scaling behavior for the second moment statistics of area-averaged rain rate on the averaging length scale $L$ as $L \rightarrow 0$. In the present work a more efficient method of estimating the model parameters is presented, and used to fit the model to the statistics of area-averaged rain rate derived from gridded radar precipitation data from TOGA COARE. Statistical properties of the data and the model predictions are compared over a wide range of averaging scales. An extension of the spectral model scaling relations to describe the dependence of the average fraction of grid boxes within an area containing nonzero rain (the "rainy area fraction") on the grid scale $L$ is also explored.
\end{abstract}

\section{Introduction}

Rainfall is a complex phenomenon involving the interplay of many physical processes in the atmosphere. Consequently, one often has to resort to a probabilistic description of the overall space-time distribution of precipitation rather than a detailed physical model of the underlying processes. In contrast to physical models, statistical models have the advantage of being conceptually economical in the sense that they generally involve fewer adjustable parameters. Moreover, they can be easily validated by comparing against data gathered over a large area and a large time period representative of a certain climatological regime. Once the model parameters are determined from a sufficiently large data set, the model provides an efficient method of describing various statistical properties of rainfall over areas with similar rain climatologies [see, e.g., Bell et al., 2001, hereafter referred to as BKK].

Rainfall, despite its apparently irregular nature, is correlated both in space and in time. Spatio-temporal variability of rain directly influences other hydrological processes, such as soil moisture transport and surface runoff. Statistical behavior of area- and/or timeaveraged rain rate at different length and time scales can be an important diagnostic for comparing actual rain behavior with predictions from global climate models. It has important consequences for the estimation of the so-called sampling error that arises in satellite measurements because of their intermittent and often incomplete coverage of a given area of the globe $[M C$ Connell and North, 1987; Bell et al., 1990; Bell and Kundu, 1996, hereafter BK96].

In this paper we present a statistical model of spacetime variability of rainfall in the mesoscale regime derived from ground based radar observations. In this model, Fourier modes of the rain intensity $R(\mathbf{x}, t)$ are treated as a set of random variables whose evolution is governed by a linear stochastic equation driven by a white noise source. This equation immediately leads to an analytical form of the rainfall power spectrum depending on just a few adjustable parameters. The 
Fourier transform of the spectrum yields the space-time covariance function of the point rain rate field, namely

$$
c\left(\mathbf{x}, t ; \mathbf{x}^{\prime}, t^{\prime}\right)=\left\langle R^{\prime}(\mathbf{x}, t) R^{\prime}\left(\mathbf{x}^{\prime}, t^{\prime}\right)\right\rangle
$$

with

$$
R^{\prime}(\mathbf{x}, t)=R(\mathbf{x}, t)-\langle R(\mathbf{x}, t)\rangle,
$$

and determines how rain statistics depend on the averaging length and time scales. Here the angle brackets represent a statistical average over a hypothetical collection or ensemble of rain fields with similar rain climatology. The model was used previously in BK96 in a study of the sampling error in preparation for the Tropical Rainfall Measuring Mission (TRMM), where it was quite successful in describing the Global Atmospheric Research Program (GARP) Atlantic Tropical Experiment (GATE) Phase I data. Here we test it with a gridded precipitation data set constructed from the radar images obtained during the Tropical Ocean Global Atmosphere - Coupled Ocean Atmosphere Response Experiment (TOGA-COARE) [Webster and Lukas, 1992]. This data set covers a longer period of observation than the GATE data set and has a finer spatial resolution. Since the data set provides "instantaneous" spatial averages on a regular rectangular cartesian grid analogous to the GATE data, it is well suited to studying the statistical behavior of the rain field averaged over various spatial scales. A parameter estimation method is described in this paper that utilizes the analytical behavior of the model and is much more convenient and robust than the method used in BK96 which involved considerable amounts of trial and error.

There exists a large class of physical rainfall models based on random processes that may be called hierarchical cluster models. These models attempt to capture the spatial and temporal organization of storm systems that cause precipitation. A general mathematical framework for such models was laid by Le Cam [1961] in which stochastic Poisson processes with suitably randomized parameters are used to describe the irregular occurrence of rain events in space and time. There is an extensive literature on this type of model [e.g., Gupta and Waymire, 1979; Cox and Isham, 1988; Smith and Krajewski, 1987; Waymire et al., 1984]. However, these models generally involve a large number of parameters whose estimation from actual rain data presents a difficult problem. For a recent account of a number of models in this category, including the problem of parameter estimation in the context of a precipitation data set obtained in the United Kingdom from the HYREX project, a large network of radar and rain gauges, see
Wheater et al. [2000]. Because of the ease of parameter estimation for real rain data sets, combined with its ability to capture the variability on many different space and time scales, we chose to consider a rainfall model based on a simple stochastic dynamical equation rather than a cluster model of the type described above.

Other stochastic dynamical rainfall models exist in the literature [North and Nakamoto, 1989; Yoo et al., 1996] which also describe the GATE spectrum with varying degrees of success. Of these the North-Nakamoto (NN) forced diffusion model is a special case of the model considered here. It describes precipitation as the diffusive spreading of rain events through a turbulent atmosphere. The Yoo-Valdés-North (YVN) model adds a second time derivative term which may help in modeling the high frequency behavior of the rainfall spectrum. However, the model has more parameters and has so far been applied only in the low wave number regime, and it is not yet clear how well the model describes rainfall statistics at the smaller spatial scales considered in this paper.

The present model is an outgrowth of previous work by Bell [1987] and Bell et al. [1990] who constructed a model to simulate the statistics of the gridded rainfall data from GATE. They introduced a gaussian random field on a discrete $4 \mathrm{~km}$ grid whose Fourier amplitudes were assumed to obey a stochastic dynamical equation similar to the one used here. A nonlinear mapping was used to generate the gridded rain rate field with a rain-rate probability distribution matching the observed lognormal distribution. Unlike that work, the present model directly describes the stochastic evolution of the point rain rate field, which can be averaged to any desired length and time scale. Moreover, it yields the full space-time covariance function in terms of a small number of model parameters. Once they are determined from data, the model allows us to relate statistics at different scales, whereas the original model of Bell [1987] was applicable only to a single spatial grid scale and, moreover, the form of the spatial covariance function was specified as an explicit function of spatial separation, in effect increasing the number of empirical parameters. However the present model pays a price for its simplicity in being restricted to describing only the second moment statistics of rain.

Our model spectrum naturally leads to power law scaling behavior of the rain statistics at small spatial scales suggesting an underlying fractal or multifractal structure of the rain field. Multifractal rainfall models have been proposed by Schertzer and Lovejoy [1987] and Gupta and Waymire [1990] extending earlier ideas 
of Lovejoy [1982], Lovejoy and Mandelbrot [1985] and Waymire [1985]. These models describe the moments of a spatially averaged rain field in terms of multifractals generated by an underlying self-similar multiplicative random cascade process [Marshak et al., 1994; Menabde et al., 1997]. The notion that the rain field is scale invariant over a broad range of spatial and temporal scales is inspired by statistical theory of turbulence and is supported by a growing body of empirical evidence [Foufoula-Georgiou and Krajewski, 1995]. An intriguing feature of the multiplicative cascade models is that they naturally lead to a lognormally distributed precipitation field. More recently, the spatial cascade models have been extended to space-time models which incorporate dynamics in the scaling regime, either through a combined scaling of the space and time variables [Marsan et al., 1996] or through a description of the time evolution of the cascade generators in terms of an autoregressive process [Over and Gupta, 1996; Seed et al., 1999]. The multifractal approach has been applied by Deidda [2000] to the GATE data to disaggregate the large scale rain and compare the observed and synthetically downscaled rain statistics at smaller scales. Empirical evidence of scaling under a combined space-time scale transformation ("dynamical scaling") has been presented by Venugopal et al. [1999]. A review of the multifractal cascade dynamics models is given in [Schertzer et al., 1997].

The paper is organized as follows: Section 2 describes the rainfall model. Here we collect a number of mathematical formulae related to the statistics of areaaveraged rain rate fields that are useful for fitting the model to data. Of particular interest to us is the way these statistics depend on the averaging length scale. Readers who are mainly interested in the applications of the model to data may wish to omit the theoretical details of the model and proceed to section 3 where we describe the gridded TOGA-COARE precipitation data set and the procedure for fitting the model to data and estimating the model parameters, returning later to section 2 for the main formulae as needed. Section 4 is devoted to a discussion of the results, focusing in particular on the scaling behavior of rain statistics at small spatial scales perhaps associated with fractal nature of the rain rate field. Some concluding remarks and directions for future work are presented in the final section. Three appendices provide mathematical details of the derivation of a number of results utilized in the main text.

\section{Modeling Rainfall Variability}

\subsection{A Spectral Model of Rain Rate Covariance}

In this subsection we give a brief account of the model. A set of results that are needed in fitting the model to data and checking some of its predictions are derived here. The underlying assumptions of the model as well as many of its salient features have already been described in detail in BK96.

\section{a. Model Basics}

As was emphasized in Bell [1987] and Bell et al. [1990], rain rate fields share with turbulent fluids the characteristic feature that the time scale over which spatial averages remain correlated depends on the size of the averaging area: the larger the averaging area, the longer the time over which the average rain rate remains correlated. This behavior must be captured by a realistic model of rainfall statistics. Although local rain statistics are expected to be affected by inhomogeneous and nonstationary directional effects such as occur, for instance, in rain bands, the model applications we envisage require a description that is valid over a large enough region and sufficiently long time for such directional effects to average out, so that the rain statistics can be treated to a good approximation as spatially homogeneous and isotropic and stationary in time. These assumptions imply that the space-time covariance function of the rain field at the points $\mathbf{x}, \mathbf{x}^{\prime}$ and times $t, t^{\prime}$ has the form

$$
c\left(\mathbf{x}, t ; \mathbf{x}^{\prime}, t^{\prime}\right)=c(\boldsymbol{\rho}, \tau)=c(\rho, \tau)
$$

where $\tau=t^{\prime}-t, \rho=\mathbf{x}^{\prime}-\mathbf{x}$ and $\rho=|\rho|$. It can be expressed as a Fourier integral

$$
c(\boldsymbol{\rho}, \tau)=\frac{1}{(2 \pi)^{3 / 2}} \int_{-\infty}^{\infty} d \omega \int d^{2} \mathbf{k} e^{i(\mathbf{k} \cdot \boldsymbol{\rho}-\omega \tau)} \tilde{c}(\mathbf{k}, \omega)
$$

where $\tilde{c}(\mathbf{k}, \omega)$ represents the (unnormalized) rain rate power spectrum. Isotropy implies $\tilde{c}(\mathbf{k}, \omega)=\tilde{c}(k, \omega)$ where $k=|\mathbf{k}|$. The spatial Fourier amplitudes

$$
a(\mathbf{k}, t)=\frac{1}{2 \pi} \int d^{2} \mathbf{x} e^{-i \mathbf{k} \cdot \mathbf{x}} R^{\prime}(\mathbf{x}, t)
$$

of the rain field fluctuation $R^{\prime}(\mathbf{x}, t)$ defined by Eq. (2) are assumed to obey the first order linear stochastic 
equation $^{1}$

$$
\frac{\partial}{\partial t} a(\mathbf{k}, t)=-\frac{1}{\tau_{k}} a(\mathbf{k}, t)+f(\mathbf{k}, t) .
$$

[One could add a term (ik.v) $a(\mathbf{k}, t)$ to Eq. (4) to take into account the effect of advection, at the expense of isotropy.] Eq. (4) represents what is sometimes referred to as a first order autoregressive [AR(1)] process [Jenkins and Watts, 1968]. It is formally analogous to the familiar Langevin equation for the velocity of a particle executing thermal Brownian motion in a viscous fluid. Here $f(\mathbf{k}, t)$ is a white noise force satisfying

$$
\left\langle f(\mathbf{k}, t) f^{\star}\left(\mathbf{k}^{\prime}, t^{\prime}\right)\right\rangle=(2 \pi) F_{0} \delta\left(\mathbf{k}^{\prime}-\mathbf{k}\right) \delta\left(t^{\prime}-t\right),
$$

where in the right hand side we have introduced the Dirac $\delta$-function, and the asterisk denotes complex conjugation. In Eq. (4) $\tau_{k}$ represents the characteristic relaxation time of $a(\mathbf{k}, t)$. As in BK96, it is assumed to have the form

$$
\tau_{k}=\frac{\tau_{0}}{\left(1+k^{2} L_{0}^{2}\right)^{1+\nu}}
$$

where $\tau_{0}$ and $L_{0}$ are characteristic time and length scales. The length scale $L_{0}$ effectively separates the long and the short wavelength regimes of the spatial fluctuations of the rain rate field. As a consequence of Eqs. (4) and (5) the power spectrum has the typical "red noise" form

$$
\tilde{c}(k, \omega)=\frac{F_{0}}{\omega^{2}+\tau_{k}^{-2}} .
$$

The analytic form of $\tau_{k}$ in (6) is suggested by the form chosen by Bell [1987] and Bell et al. [1990] who, in turn, were motivated by the empirical results of Laughlin [1981] from GATE data. As already noted, it contains the North-Nakamoto model as a special case with $\nu=0$. It may be pointed out that in the original work of Bell [1987] and Bell et al. [1990], the form of the spatial covariance function was separately introduced from an empirical fit, whereas in the present model it is implicitly contained in the analytic form of $\tau_{k}$.

Because of the fractional exponent $\nu$, the implied stochastic dynamical equation governing the evolution of the rain rate field $R(\mathbf{x}, t)$ now takes the form of an

${ }^{1} \mathrm{~A}$ more general possibility is an equation of the form

$$
\frac{\partial}{\partial t} a(\mathbf{k}, t)=-\int d^{2} \mathbf{k}^{\prime} \frac{1}{\tau_{k k^{\prime}}} a\left(\mathbf{k}^{\prime}, t\right)+f(\mathbf{k}, t),
$$

which allows coupling among different Fourier modes. integro-differential equation with an associated nonlocal character rather than a pure differential equation as in the case of NN and YVN models. It can also be formally expressed in terms of fractional order derivatives [see, e.g., Benson et al., 2000]. Physically it can be thought of as representing a generalized diffusion process by which rain moves through a turbulent atmosphere. In particular, the negative values of $\nu$ required to fit the data sets studied here appear to better describe the high variability of rain rates at small spatial scales - an important factor in extrapolating statistics to rain gauge scales.

The four model parameters $F_{0}, \tau_{0}, L_{0}$ and $\nu$ characterize the second moment statistics. No specific assumption about the actual probability distribution for the random variable $R(\mathbf{x}, t)$ is introduced here. It should be noted however that the model of rain statistics remains incomplete without such an assumption, since the higher order moments cannot be represented within the model. Observationally, area averaged rain rates seems to be approximately lognormally or gamma distributed, at least on smaller space and time scales.

\section{b. Spatial Covariance Function}

Substituting the explicit form of the model spectrum in (3) and performing the integral over $\omega$ and the angular integral in the $\mathbf{k}$-plane yields

$$
c(\rho, \tau)=\int_{0}^{\infty} d k k J_{0}(k \rho) c(k, \tau),
$$

where $J_{0}(x)$ is the Bessel function of order zero, and

$$
c(k, \tau)=\sqrt{\pi / 2} F_{0} \tau_{k} e^{-|\tau| / \tau_{k}}
$$

represents the lagged covariance of $a(\mathbf{k}, t)$ defined by the equation

$$
\left\langle a(\mathbf{k}, t) a^{\star}\left(\mathbf{k}^{\prime}, t^{\prime}\right)\right\rangle=(2 \pi) c(k, \tau) \delta\left(\mathbf{k}^{\prime}-\mathbf{k}\right),
$$

and is the inverse temporal Fourier transform of $\tilde{c}(k, \omega)$. At zero lag $(\tau=0)$, carrying out the remaining $k$ integration in (8), the spatial covariance of the point rain rate can be expressed in the simple closed form

$$
c(\rho, \tau=0)=\gamma_{0} C_{\nu}\left(\rho / L_{0}\right),
$$

where, for convenience, $\gamma_{0}$ is defined by the relation

$$
F_{0}=\sqrt{2 / \pi} \Gamma(1+\nu)\left(L_{0}^{2} / \tau_{0}\right) \gamma_{0},
$$

$\Gamma(z)=\int_{0}^{\infty} t^{z-1} e^{-t} d t$ is the Euler $\Gamma$-function, and

$$
C_{\nu}(z)=(z / 2)^{\nu} K_{\nu}(z)
$$


is a function related to the modified Bessel function $K_{\nu}(z)$. For $z \ll 1, C_{\nu}(z)$ has the behavior

$C_{\nu}(z)=\frac{1}{2} \Gamma(\nu)+\frac{1}{2} \Gamma(-\nu)\left(\frac{z}{2}\right)^{2 \nu}+O\left(z^{2+2 \nu}\right)+O\left(z^{2}\right)$.

For $\nu>0$, the first term dominates and $C_{\nu}(z) \rightarrow \frac{1}{2} \Gamma(\nu)$ as $z \rightarrow 0$, and the variance of the point rain rate $c(0,0)=\frac{1}{2} \gamma_{0} \Gamma(\nu)$ is finite. However, as we shall see, like the GATE data, the TOGA-COARE data requires $\nu<0$, and the covariance function exhibits a power law singularity

$$
c(\rho, 0) \sim \frac{1}{2} \gamma_{0} \Gamma(|\nu|)\left(\rho / 2 L_{0}\right)^{-2|\nu|}
$$

as the separation $\rho \rightarrow 0$. This remarkable singular behavior can be traced to the $k$-dependence of the characteristic time scale $\tau_{k}$ governing the decay rate of the Fourier mode $a(\mathbf{k}, t)$ : For the long wavelength modes $(k \approx 0) \tau_{k}$ approaches a constant value $\tau_{0}$, whereas for the short wavelength modes $\left(k \gg 1 / L_{0}\right) \tau_{k}$ behaves like $k^{-2(1-|\nu|)}$. Note that for values of the exponent $\nu$ in the range $-1<\nu<0$ (as seems to be the case for both GATE and TOGA-COARE data sets), $\tau_{k} \rightarrow 0$ as $k \rightarrow \infty$. For the NN model $(\nu=0)$ the singularity in $c(\rho, 0)$ weakens to become logarithmic.

Although the point variance is infinite in the model, this is not necessarily inconsistent with experience since observations always involve smoothing of the point rain rate field in space and/or time, and variances of smoothed rain field are finite in the model.

\section{c. Statistics of Area-Averaged Rain Fields}

Next, we turn to the space-time covariance function of rain rate averaged over a square area $A=L^{2}$,

$$
\mathcal{R}_{\mathcal{A}}(t)=\frac{1}{A} \int_{\mathcal{A}} d^{2} \mathbf{x} R(\mathbf{x}, t)
$$

at lag $\tau$ namely,

$$
\mathcal{C}_{\mathcal{A A}^{\prime}}(\mathbf{s}, \tau)=\left\langle\mathcal{R}_{\mathcal{A}}^{\prime}(t) \mathcal{R}_{\mathcal{A}^{\prime}}^{\prime}(t+\tau)\right\rangle
$$

where $\mathcal{A}, \mathcal{A}^{\prime}$ are two $L \times L$ boxes whose centers are separated by $\mathbf{s}$ and the prime on the rain rate variables denotes deviation from the mean, as in Eq. (2). Starting from the formula

$$
\mathcal{C}_{\mathcal{A} \mathcal{A}^{\prime}}(\mathbf{s}, \tau)=\frac{1}{A^{2}} \int_{\mathcal{A}} d^{2} \mathbf{x} \int_{\mathcal{A}^{\prime}} d^{2} \mathbf{x}^{\prime} c\left(\mathbf{s}+\mathbf{x}^{\prime}-\mathbf{x}, \tau\right)
$$

the spectral model yields at zero lag the expression

$$
\mathcal{C}_{\mathcal{A A}^{\prime}}(\mathbf{s}, 0)=\gamma_{0} \int_{-1}^{1} \int_{-1}^{1} d \xi_{1} d \xi_{2}\left(1-\left|\xi_{1}\right|\right)\left(1-\left|\xi_{2}\right|\right)
$$

$$
\times \quad C_{\nu}\left(\left|\boldsymbol{\xi}+\frac{\mathbf{s}}{L}\right| \frac{L}{L_{0}}\right) .
$$

See Appendix A in BK96 for details of the derivation. Upon setting $s=0$ in Eq. (16), the variance of the areaaveraged rain rate in a grid box of area $A=L^{2}$ is given by

$$
\sigma_{A}^{2} \equiv \sigma^{2}(L)=4 \gamma_{0} G\left(\nu ; L / L_{0}\right),
$$

where $G(\nu ; z)$ is the double integral

$$
\begin{aligned}
G(\nu ; z) & =\int_{0}^{1} \int_{0}^{1} d \xi_{1} d \xi_{2}\left(1-\xi_{1}\right)\left(1-\xi_{2}\right) \\
& \times C_{\nu}\left(z \sqrt{\xi_{1}^{2}+\xi_{2}^{2}}\right)
\end{aligned}
$$

The power law singularity of the spatial covariance of the point rain rate as a function of separation leads to a simple scaling behavior of the statistics of area averaged rain with the size of the area. Using the first two terms of the expansion (14) in Eq. (19), which dominate as long as $-1<\nu<0$, we have

$$
G(\nu ; z) \approx \frac{1}{8} \Gamma(-|\nu|)+\frac{1}{2} \Gamma(|\nu|) H(\nu)\left(\frac{z}{2}\right)^{-2|\nu|},
$$

where $H(\nu)$ denotes the integral

$$
H(\nu)=\int_{0}^{1} \int_{0}^{1} d \xi_{1} d \xi_{2}\left(1-\xi_{1}\right)\left(1-\xi_{2}\right)\left(\xi_{1}^{2}+\xi_{2}^{2}\right)^{\nu} .
$$

Hence, for small $L$ (more precisely $L \ll L_{0}$ ), $\sigma^{2}(L)$ can be expressed in the form

$$
\sigma^{2}(L) \approx a_{0}+b_{0} L^{-2|\nu|},
$$

where $a_{0}$ and $b_{0}$ are constants given by

$$
\begin{gathered}
a_{0}=\frac{1}{2} \gamma_{0} \Gamma(-|\nu|), \\
b_{0}=2 \gamma_{0} \Gamma(|\nu|) H(\nu)\left(2 L_{0}\right)^{2|\nu|} .
\end{gathered}
$$

Thus the model predicts that as $L \rightarrow 0$, the variance of area-averaged rain rate $\sigma^{2}(L)$ exhibits a power law singularity:

$$
\sigma^{2}(L) \sim L^{-2|\nu|} .
$$

\section{d. Correlation Length and Time Scales}

The lagged correlation function

$$
\Phi_{\mathcal{A A}^{\prime}}(\mathbf{s}, \tau)=\frac{\mathcal{C}_{\mathcal{A A}^{\prime}}(\mathbf{s}, \tau)}{\sigma_{A}^{2}}
$$


is a rapidly decreasing function of the separation s. The model correlation function is markedly nonexponential. Its fall-off rate is conveniently characterized by the "integral correlation length" $\Lambda_{\mathrm{int}}(L)$ [which would be the (1/e)-folding distance for an exponentially decaying correlation function] defined in the isotropic case through the relation

$$
\Lambda_{\mathrm{int}}^{2}(L)=\int_{0}^{\infty} d s s \Phi_{\mathcal{A} \mathcal{A}^{\prime}}(\mathbf{s}, \tau=0) .
$$

It is related to the model spectrum through

$$
\Lambda_{\mathrm{int}}^{2}(L)=\frac{1}{\sigma^{2}(L)} c(\mathbf{k}=0, \tau=0),
$$

which upon using Eq. (18) reduces to

$$
\Lambda_{\text {int }}^{2}(L)=\frac{1}{4} \Gamma(1+\nu) L_{0}^{2} / G\left(\nu ; L / L_{0}\right) .
$$

From Eq. (20) it then follows that $\Lambda_{\text {int }}(L)$ vanishes as $L^{|\nu|}$ as $L \rightarrow 0$.

The case $\nu=-1$ presents a particularly simple and analytically tractable special case of our spectral model. In this case the spatial covariance of the point rain rate field satisfies $c(\rho, 0) \propto \delta(\rho)$. As a consequence, the correlation between the rain rates in disjoint grid boxes vanish. The integral correlation length $\Lambda_{\text {int }}(L)$ is simply equal to the box size $L$ and the variance of areaaveraged rain rate $\sigma^{2}(L)$ is proportional to $1 / L^{2}$.

We can also define an integral autocorrelation time $\tau_{\text {int }}(L)$ (which would be the $1 / e$-folding time for an exponentially decreasing autocorrelation function) through the formula

$$
\tau_{\mathrm{int}}(L)=\int_{0}^{\infty} d \tau \Phi_{\mathcal{A \mathcal { A }}}(\mathbf{s}=0, \tau) .
$$

It can be expressed in terms of the Fourier transform $\tilde{\mathcal{C}}_{A}(\omega)$ of the lagged autocovariance function $\mathcal{C}_{\mathcal{A} A}(\mathbf{s}=0, \tau)$ as

$$
\tau_{\text {int }}(L)=\sqrt{\frac{\pi}{2}} \frac{\tilde{\mathcal{C}}_{A}(\omega=0)}{\sigma_{A}^{2}} .
$$

Evaluation of $\tilde{\mathcal{C}}_{A}(0)$ (see Appendix A) yields

$$
\tau_{\text {int }}(L)=\tau_{0} \frac{\Gamma(1+\nu)}{\Gamma(2+2 \nu)} \frac{G\left(1+2 \nu ; L / L_{0}\right)}{G\left(\nu ; L / L_{0}\right)} .
$$

The values of $\nu$ that best describe rain data in fact appear to lie in the narrower range $-1 / 2<\nu<0$ (rather than the range $-1<\nu<0$ considered above). The numerator of Eq. (31) is then finite and consequently the model predicts that $\tau_{\text {int }}(L) \rightarrow 0$ as $L^{2|\nu|}$ in the linit $L \rightarrow 0$. In other words, the time scale for fluctuations of average rain rate in a box of area $L^{2}$ gets smaller with decreasing box size.

\subsection{Extension to Other Rain Statistics}

The spectral model presented above makes a number of predictions about the change of rain statistics with scale. With a few additional assumptions these predictions can be shown to have interesting consequences for the asymptotic behavior of the "conditional" statistics of rain as well. We now turn to a brief description of these statistics.

Consider remote sensing measurements of precipitation over a given area and time period and the resulting rain maps gridded on an $L \times L$ square grid. The ensemble mean rain rate $\langle R\rangle$ is estimated as the average of all the data within the data set, and is independent of the resolution scale $L$. However, other statistical properties of the field, such as the fraction of grid boxes containing nonzero rain ("the rainy area fraction") $p(L)$ and the variance of the grid-box-averaged rain rate $\sigma^{2}(L)$, already considered, depend nontrivially on $L$. The quantity $p(L)$ representing the probability of nonzero rain in a grid box is a measure of the spatial intermittency of rain at the scale $L$. Two other related statistics are the mean $r_{c}(L)$ and the variance $\sigma_{c}^{2}(L)$ of the grid-boxaveraged rain rate conditional on the box containing nonzero rain. These are related (see, e.g., BKK, Appendix A) through

$$
\begin{gathered}
\langle R\rangle=p(L) r_{c}(L), \\
\sigma^{2}(L)=p(L)\left[\sigma_{c}^{2}(L)+r_{c}(L)^{2}\right]-\langle R\rangle^{2} .
\end{gathered}
$$

It is convenient to define the dimensionless ratio

$$
\mu \equiv \mu(L)=\sigma_{c}(L) / r_{c}(L) .
$$

Using Eq. (32), Eq. (33) can then be written as

$$
1+\frac{\sigma^{2}(L)}{\langle R\rangle^{2}}=\frac{1+\mu^{2}}{p(L)} .
$$

Based on analysis of rain gauge data from Darwin, Australia and Melbourne, Florida and radar data from GATE, Short et al. [1993] have suggested that $\mu$ is relatively constant over a range of length scales, averaging times, types of data (radar or rain gauge) and rain climates. In particular, $\mu$ is insensitive to the averaging length scale $L$. This was also found by BKK for SSM/I rain data.

If the quantity $\mu(L)$ remains finite as $L \rightarrow 0$, then Eq. (35) together with the asymptotic behavior (22) predicts that $p(L)$ would vanish as $L^{2|\nu|}$ in this limit. The limiting behavior $p(L) \rightarrow 0$ as $L \rightarrow 0$ in turn implies that the instantaneous point rain rate field $R(\mathbf{x}, t)$ vanishes almost everywhere, i.e., everywhere except a 
set of measure zero - a familiar property of spatial fractals. We shall return to this in greater detail in section 4 when we present the results of our data analysis.

\section{Testing the Model Predictions with Ground based Radar Data}

\subsection{The TOGA-COARE dataset}

The radar data we use to test the spectral model described above were collected during TOGA- COARE [Webster and Lukas, 1992] carried out in the Western Pacific equatorial warm pool during the period November 1992 to February 1993. The measurements were made with a pair of calibrated Doppler radars (labeled TOGA and MIT) on board two research ships located near the center of the Intensive Flux Array (IFA) - a region in the shape of a quadrilateral, roughly centered at $2^{\circ} \mathrm{S}, 156^{\circ} \mathrm{E}$. Reliable rain rate estimates were provided out to a distance $145 \mathrm{~km}$ from each radar. The fields of view (FOVs) of the two radars overlapped substantially when they were both operating. The measured radar reflectances $Z$ were converted into average surface rain rates on a $2 \mathrm{~km}$ spatial grid using an empirical $Z-R$ relation. The data set is described by Short et al. [1997]. The total 101-day period of observation was divided into three "monthly" cruises covering the periods November 11 to December 10 1992, December 151992 to January 181993 and January 23 to February 231993 designated respectively as Cruises 1,2 and 3. Rain maps were available roughly at intervals of ten minutes with occasional long gaps during which data from one or both of the radars were missing.

\subsection{Statistical Analysis of the Data}

The gridded data set obtained from the TOGACOARE observations yields estimates of the various area-averaged rain statistics introduced previously, allowing one to test the model predictions. We now briefly describe how these statistics are obtained from the data.

The statistics are derived using rain estimates from square areas $128 \times 128 \mathrm{~km}^{2}$ centered at the location of each of the two ships. The locations of the areas changed slowly with time because of the slight drift of the ships during the month-long experiments. However, the effect of this on the statistics, if any, is expected to be negligible. Each area is subdivided into boxes $L \times L$ in size, with $L=2^{n} \mathrm{~km}(n=1,2, \ldots, 7)$, and the quantities $\langle R\rangle, \sigma^{2}(L), p(L), r_{c}(L)$ and $\sigma_{c}(L)$ defined in the previous section are computed for each $L$ from the data. Only those boxes in a rain map were used for which at least $95 \%$ of the box had valid data. This was necessary since the radar images frequently had data missing along a radial line emanating from the center of the circular FOV where the radar was located. These dropouts affected the grid boxes located close to the origin through which the radial line passed, especially at the smaller grid length scales $L=2,4,8$ and $16 \mathrm{~km}$.

For comparison purposes, we also computed the same spatial statistics for the GATE Phase I (1716 images) and Phase II (1512 images) data from the $280 \mathrm{~km}$ box centered at the location of the ship. For GATE data the basic grid size is $4 \mathrm{~km}$.

We determined the integral correlation time $\tau_{\text {int }}(L)$ for each $L$ as follows. Mean rain rate time series were constructed for each $L \times L$ box within the selected areas for the three cruises. Again only boxes with at least $95 \%$ of the area containing data were included in the time series in order to contend with the data gaps mentioned above. The lagged autocovariance function $\mathcal{C}_{\mathcal{A A}}(0, \tau)$ averaged over all boxes in the chosen areas was computed for each $L$ at lags $\tau$ of multiples of $10 \mathrm{~min}$.

As an example, the lagged autocorrelation function

$$
\Phi_{L}(\tau) \equiv \Phi_{\mathcal{A} \mathcal{A}}(0, \tau)
$$

for $L=128 \mathrm{~km}$ for the six data sets are shown in Figure 1. For each data set a numerical estimate of the time integral $\tau_{\text {int }}(L)=\int_{0}^{\infty} \Phi_{L}(\tau) d \tau$ was obtained. This is discussed in more detail later.

The next subsection describes how the parameters $\gamma_{0}, L_{0}, \nu$ and $\tau_{0}$ are determined by fitting the data to the statistics of gridded averages predicted by the model.

\subsection{Estimation of the Model Parameters}

The parameters $\gamma_{0}, L_{0}$ and $\nu$ determine the spatial statistics at zero lag. They are estimated by fitting the variance $\sigma^{2}(L)$ to the asymptotic formula (22) valid for small $L$. The fit determines the exponent $\nu$ and the coefficients $a_{0}$ and $b_{0}$. Eq. (23) determines the parameter $\gamma_{0}$ and Eq. (24) then determines $L_{0}$. The procedure was carried out for the TOGA-COARE as well as both GATE Phase I and Phase II data sets.

Once $\nu$ and $L_{0}$ are determined, an estimate of the parameter $\tau_{0}$ for each data set can be obtained by averaging over the values individually computed by solving (31) for $\tau_{0}$ using the values of $\tau_{\text {int }}(L)$ for various box sizes $L$. However, since there is only a finite length $T$ of data available, instead of $\tau_{\text {int }}(L)$ one can only obtain an estimate

$$
\hat{\tau}_{\text {int }}\left(L, \tau_{0} ; \tau_{\max }\right)=\int_{0}^{\tau_{\max }} \Phi_{L}(\tau) d \tau
$$




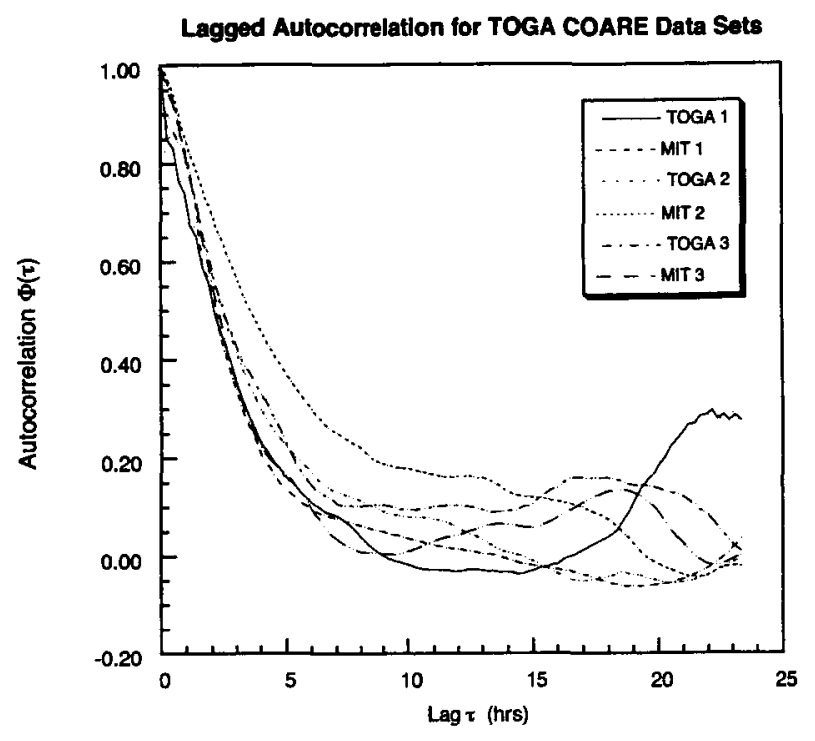

Figure 1. Plot of lagged autocorrelation functions $\Phi_{L}(\tau)$ for $L=128 \mathrm{~km}$ square boxes centered at the location of the TOGA and MIT radars for each of the three cruises.

with a finite cut-off $\tau_{\max }<T$ imposed on (29). In principle, we could solve (36) for $\tau_{0}$ using the model from the data-derived value of $\hat{\tau}_{\text {int }}$ for a chosen cut-off $\tau_{\max }$. In practice this is difficult, since the model-predicted $\tau_{0}$-dependence of the function $\hat{\tau}_{\text {int }}\left(L, \tau_{0} ; \tau_{\max }\right)$ is nonlinear and complex to calculate. Instead, we adopt an iterative method described next.

There is an uncertainty inherent in choosing $\tau_{\max }$ in order to get the "best estimate" of $\tau_{0}$. It arises because the tail of the autocorrelation function suffers from a combined effect of statistical noise and possible secular variations of the rainfall pattern over longer time scales not represented in our spectral model. The choice of the cut-off is guided by several considerations. On the one hand, $\tau_{\max }$ should be large enough so that the contribution of the tail in the integral (29) is small. Some guidance on an acceptable value of $\tau_{\max }$ is provided by the model's prediction of this contribution. However, the empirical lagged autocorrelation suffers from sampling error because it is obtained from a finite time series of length $T \approx 30$ days. The cut-off $\tau_{\max }$ must therefore satisfy the condition $\tau_{\max } \ll T$. Also $\tau_{\max }$ should be chosen to be small enough so that the effect of slow secular modulations of the lagged autocorrelation function caused by physical phenomena such as a diurnal cycle or various atmospheric waves, which are not accounted for in our simple model spectrum, is minimized. Evidence of such longer time scales was most obvious in the
Cruise 2 MIT and the Cruise 3 TOGA radar data sets. These modulations can probably be attributed to various large scale atmospheric waves known to influence rainfall in the equatorial Pacific. We shall revisit some of these issues in the next section. Keeping these factors in mind, we chose a relatively small cut-off $\tau_{\max }=12$ hours with the implicit assumption that the spectral model accurately describes the observed correlations up to the lag $\tau=\tau_{\max }$.

Next, we turn to the problem of estimation of $\tau_{0}$. Using (36), Eq. (29) for the integral correlation time $\tau_{\text {int }}(L) \equiv \tau_{\text {int }}\left(L, \tau_{0}\right)$ can be expressed as the sum

$$
\tau_{\text {int }}\left(L, \tau_{0}\right)=\hat{\tau}_{\text {int }}\left(L, \tau_{0} ; \tau_{\max }\right)+\int_{\tau_{\max }}^{\infty} \Phi_{L}(\tau) d \tau
$$

If the left-hand side of (37) were known, Eq. (31) would immediately yield $\tau_{0}$. However, while the first term on the right-hand side of (37) is obtained from data, the second term can be calculated from the model only when $\tau_{0}$ is known. We therefore solve Eq. (37) iteratively: to begin the process, we obtain a first guess for $\tau_{0}$ by ignoring the contribution of the integral; for all the following steps of the iteration we evaluate the integral on the right-hand side using the model $\Phi_{L}(\tau)$ with the value of $\tau_{0}$ obtained from the previous step. For ease of computation, we replace the square box by a circular disk of equal area. This approximation proved to be accurate enough for our purposes. Appendix B gives some details of the model calculations for a circular area. In practice, for the TOGA-COARE data, the correction to $\tau_{0}$ due to the introduction of a cutoff is of order $5 \%$, and it was unnecessary to go beyond the first iteration.

The iterative method applied to GATE Phase I data with $\tau_{\max }$ chosen to be $18 \mathrm{~h}$ gives an estimate of $\tau_{0}$ within about $1 \%$ of what was reported in BK96 from a direct fit to the lagged autocorrelation function.

\section{Results and Discussion}

The full set of parameter values for which the model best fits the TOGA-COARE data are given in Table 1, along with the corresponding values for GATE Phase I determined in BK96 using a somewhat less exact and more arduous trial-and-error curve fitting method instead of the analytical method adopted here.

\subsection{Model Parameters $\nu, L_{0}$ and $\gamma_{0}$}

Table 1 summarizes the TOGA-COARE model parameters. We see that the values of the power law exponent $\nu$ range between about -0.21 to -0.34 indicating 
Table 1. Parameter Values for Rain-Rate Covariance Model.

\begin{tabular}{lccccc}
\hline \multicolumn{1}{c}{ Dataset } & $\gamma_{0}\left(\mathrm{~mm}^{2} \mathrm{~h}^{-2}\right)$ & $\nu$ & $L_{0}(\mathrm{~km})$ & $\tau_{0}(\mathrm{~h})$ & $\langle R\rangle\left(\mathrm{mm} \mathrm{h}^{-1}\right)$ \\
\hline GATE Phase I & 1.0 & -0.11 & 104. & 13.0 & 0.492 \\
TOGA Cruise 1 & 0.067 & -0.335 & 94.06 & 6.8 & 0.139 \\
MTT Cruise 1 & 0.086 & -0.297 & 73.89 & 5.8 & 0.134 \\
TOGA Cruise 2 & 0.616 & -0.239 & 53.81 & 5.0 & 0.352 \\
MTT Cruise 2 & 0.206 & -0.205 & 70.40 & 8.2 & 0.229 \\
TOGA Cruise 3 & 0.127 & -0.290 & 61.04 & 5.2 & 0.155 \\
MTT Cruise 3 & 0.180 & -0.259 & 64.94 & 4.5 & 0.200 \\
\hline
\end{tabular}

Parameters for the model spectrum obtained from fits to radar data from Phase I of GATE and from the ships MIT and TOGA during the three TOGA-COARE cruises. Average rain rate for each dataset is given in the last column.

a much stronger singular behavior than was found for the GATE Phase I data $(\nu=-0.11)$. The characteristic time $\tau_{0}$ lies in the range of roughly 4.5 to 8.2 hours, considerably shorter than the GATE Phase I value $\tau_{0} \approx 13$ hours. The characteristic length $L_{0}$ ranges between about 54 and $94 \mathrm{~km}$ indicating a somewhat sharper fall-off of the spatial correlations compared to GATE Phase I for which $L_{0}=104 \mathrm{~km}$. The results exhibiting the quality of the fit are shown in Figure 2 for the TOGA and MIT radars. It is seen that in most cases the fit is quite good up to grid size $L=64 \mathrm{~km}$, beyond which the approximation on which Eq. (22) is based is expected to break down. Our results seem to be consistent with the model prediction that the variance $\sigma^{2}(L)$ has a power law behavior $\sigma^{2}(L) \sim L^{-2|\nu|}$ as $L \rightarrow 0$. Robustness of the parameterization was checked by comparing the variance of area-averaged rain rate $\left(\sigma_{A}^{2}\right)_{\text {th }}$ predicted by the exact formula (18) for an area $A=L^{2}$ with $L=128 \mathrm{~km}$ against the observed variance $\left(\sigma_{A}^{2}\right)_{\text {obs }}$ for the $128 \mathrm{~km}$ box centered at the two ship locations. Table 2 shows the comparisons, which are quite good.

For GATE data we find that for $L$ between $4 \mathrm{~km}$ and $56 \mathrm{~km}$, the variance $\sigma^{2}(L)$ is quite accurately fitted (not shown here) by the formulas

$$
\sigma^{2}(L)= \begin{cases}17.3 L^{-0.24}-4.66 \mathrm{~mm}^{2} \mathrm{~h}^{-2}, & \text { Phase I, } \\ 15.0 L^{-0.38}-1.92 \mathrm{~mm}^{2} \mathrm{~h}^{-2}, & \text { Phase II. }\end{cases}
$$

Equations (22) - (24) yield the parameter values

$$
\gamma_{0}=1.02 \mathrm{~mm}^{2} \mathrm{~h}^{-2}, \nu=-0.12, L_{0}=93.8 \mathrm{~km}
$$

for Phase I and

$$
\gamma_{0}=0.63 \mathrm{~mm}^{2} \mathrm{~h}^{-2}, \nu=-0.19, L_{0}=82.7 \mathrm{~km}
$$

for Phase II. [The original GATE Phase I parameters listed in Table 1 lead to the asymptotic formula $\sigma^{2}(L)=$ $16.80 L^{-0.22}-4.89 \mathrm{~mm}^{2} \mathrm{~h}^{-2}$ (see BK96 Appendix A), whose coefficients and the power law exponent are well within the $95 \%$ confidence interval of the corresponding quantities in the empirical least squares fit given above.]

We also explored the dependence of the model parameters on the mean rain rate $\langle R\rangle$. While no systematic dependence could be discerned for $L_{0}, \tau_{0}$ and $\nu$, the strength parameter $\gamma_{0}$ was found to depend on $\langle R\rangle$ according to the simple formula

$$
\gamma_{0}=\kappa\langle R\rangle^{2},
$$

where $\kappa$ is a dimensionless constant. The fit in Figure 3 , which also includes points corresponding to GATE Phase I $\left(\langle R\rangle=0.492 \mathrm{~mm} \mathrm{~h}^{-1}\right)$ and GATE Phase II $\left(\langle R\rangle=0.386 \mathrm{~mm} \mathrm{~h}^{-1}\right)$, yields a value $\kappa \approx 4.33$.

\subsection{Model Parameter $\tau_{0}$}

Next, we turn to a discussion of the dependence of the integral correlation time $\tau_{\text {int }}(L)$ on the spatial averaging scale $L$. The plots in Fig. 4 show reasonable agreement between the values of $\tau_{\text {int }}(L)$ determined from data and the corresponding theoretical predictions computed for various box sizes $L$ using the model parameters. Linearity of the $\log -\log$ plot for small $L$ confirms the power law dependence $\tau_{\text {int }}(L) \sim\left(L / L_{0}\right)^{2|\nu|}$ as $L \rightarrow 0$ expected from our model. In BK96 it was found that the integral correlation time estimates for the GATE Phase I is described quite well by the power law form $\tau_{\text {int }}(L)=0.67 L^{0.49} \mathrm{~h}$. Again the exponent of the power law is rather different from the expected value 0.22 obtained from the spectral model fit. However, it 
Table 2. Comparison between theoretical and observed values of $\sigma_{A}^{2}$ for $L=128 \mathrm{~km}$.

\begin{tabular}{lcc}
\hline \multicolumn{1}{c}{ Dataset } & $\left(\sigma_{A}^{2}\right)_{\mathrm{th}}\left(\mathrm{mm}^{2} \mathrm{~h}^{-2}\right)$ & $\left(\sigma_{A}^{2}\right)_{\text {obs }}\left(\mathrm{mm}^{2} \mathrm{~h}^{-2}\right)$ \\
\hline TOGA Cruise 1 & 0.107 & 0.116 \\
MIT Cruise 1 & 0.093 & 0.105 \\
TOGA Cruise 2 & 0.399 & 0.371 \\
MIT Cruise 2 & 0.176 & 0.174 \\
TOGA Cruise 3 & 0.107 & 0.119 \\
MIT Cruise 3 & 0.155 & 0.169 \\
\hline
\end{tabular}

Comparison between values of the variance of area-averaged rain rate $\sigma_{A}^{2}$ computed from the model using Eq. (18) and the parameters listed in Table 1 for $L=128 \mathrm{~km}$ and from the data for two $128 \mathrm{~km}$ boxes centered at each of the two ship locations.

was also found there that while the model captures the overall decay of the autocorrelation function $\Phi_{L}(\tau)$, it substantially underestimates the correlation at smaller lags, indicating a departure from the model spectrum for a first order process. Indeed, it was shown by Bell and Reid [1993] that this behavior could be accounted for by going over to a second order model, which of course introduces more adjustable parameters.

A number of caveats in the estimates of $\tau_{0}$ should be pointed out. A fundamental uncertainty in the value of $\tau_{\text {int }}(L)$ arises from the fact that it must be estimated from a time series of finite length $T$ which in our case is of order 30 days. It is known [Bell, 1980] that if the true autocorrelation function of a normally distributed random variable has an exponentially decaying form $\Phi_{L}(\tau)=\exp \left(-\tau / \tau_{\text {int }}\right)$ then the integral correlation $\hat{\tau}_{\text {int }}$, defined by Eq. (35) with a finite cut-off $\tau_{\max }$, has variance

$$
\sigma^{2}\left[\hat{\tau}_{\mathrm{int}}\right]=4\left(\tau_{\max } / T\right) \hat{\tau}_{\mathrm{int}}^{2},
$$

where it is assumed that $\tau_{\text {int }}(L) \ll \tau_{\max } \ll T$. A proof of Eq. (39) is given in Appendix C. Thus, in order to reduce the statistical uncertainty in the estimate, $\tau_{\max }$ should be chosen to be as small as possible but large enough to include the entire range over which $\Phi_{L}(\tau)$ differs appreciably from zero. In our case, if we choose $\tau_{\max } \approx 12 \mathrm{hrs}$, then it follows that the $2 \sigma$ uncertainty in the estimate of $\tau_{\text {int }}(L)$ obtained from a single time series could, in fact, be as large as $0.5 \hat{\tau}_{\text {int }}$. It is to be noted that, strictly speaking, this estimate holds only for a gaussian random variable undergoing a linear stochastic dynamical process with an exponentially decaying autocorrelation function. However, the probability distribution of rain rate variable is markedly nongaussian and the autocorrelation possesses a much more slowly decaying tail. These factors can lead to an uncertainty larger than what is predicted by Eq. (39). On the other hand, for the smaller grid boxes of size $L=2^{n} \mathrm{~km}$ with $n \leq 6$, the actual uncertainty should be considerably lower than the estimate (39) since the autocovariances were obtained by averaging over the results for the time series for the $4^{7-n}$ boxes. These time series are, however, not completely independent of one another, because of the spatial correlation of the rain rate in a box with others in its vicinity, and an exact estimate of the uncertainty would be difficult to obtain.

\subsection{Conditional Statistics and the Spectral Model}

The spectral model described in section 2 allows one to quantify how the statistics of area-averaged rain rate depend on the averaging scale $L$. These statistical quantities, such as the variance $\sigma^{2}(L)$ and the integral correlation length and time scales, pertain to variability of the rain field including its zeroes, i.e., the region where it is not actually raining. However, unlike most random variables in nature, the instantaneous rain rate has a finite nonzero probability of having the value zero, in accordance with common experience - namely, that it is not raining in most places most of the time. Mathematically this is expressed by the property that the probability density function for the area-averaged instantaneous rain rate $\mathcal{R}_{\mathcal{A}}(t)$ defined by (1) has a $\delta$-function at zero followed by an (approximately lognormal) continuum [Kedem et al., 1990]. The probability $p(L)$ for the value $\mathcal{R}_{\mathcal{A}} \neq 0$ is expected to have nontrivial $L$-dependence and is an important statistic of the gridded rain field. 
Variance as function of area for TOGA COARE
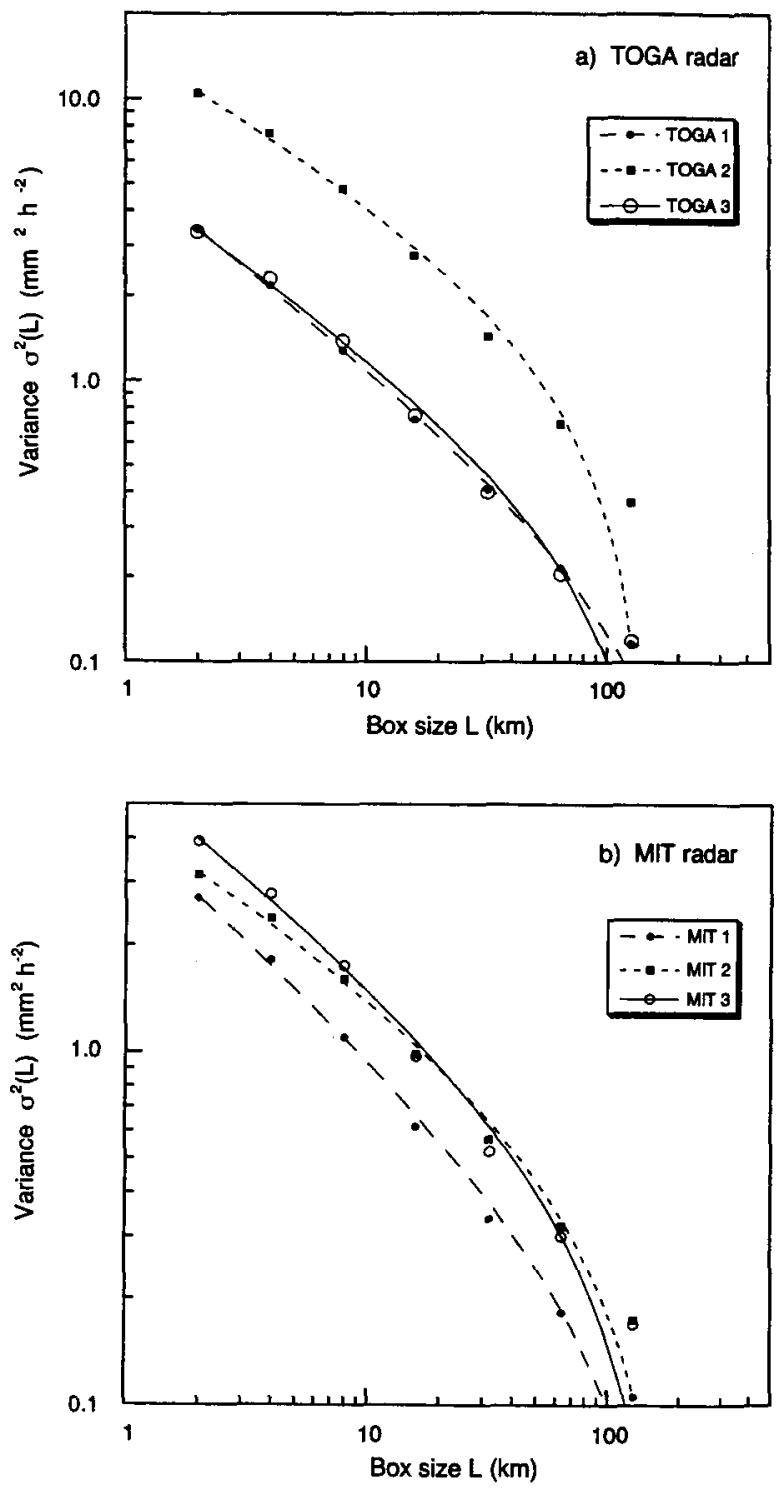

Figure 2. Comparison between the observed variance of area-averaged rain rate $\sigma^{2}(L)$ for the TOGA-COARE data and the corresponding spectral model predictions for various box sizes $L$ ranging from $2 \mathrm{~km}$ to $128 \mathrm{~km}$ using Eqs. (22)-(24) and the parameter values listed in Table 1.

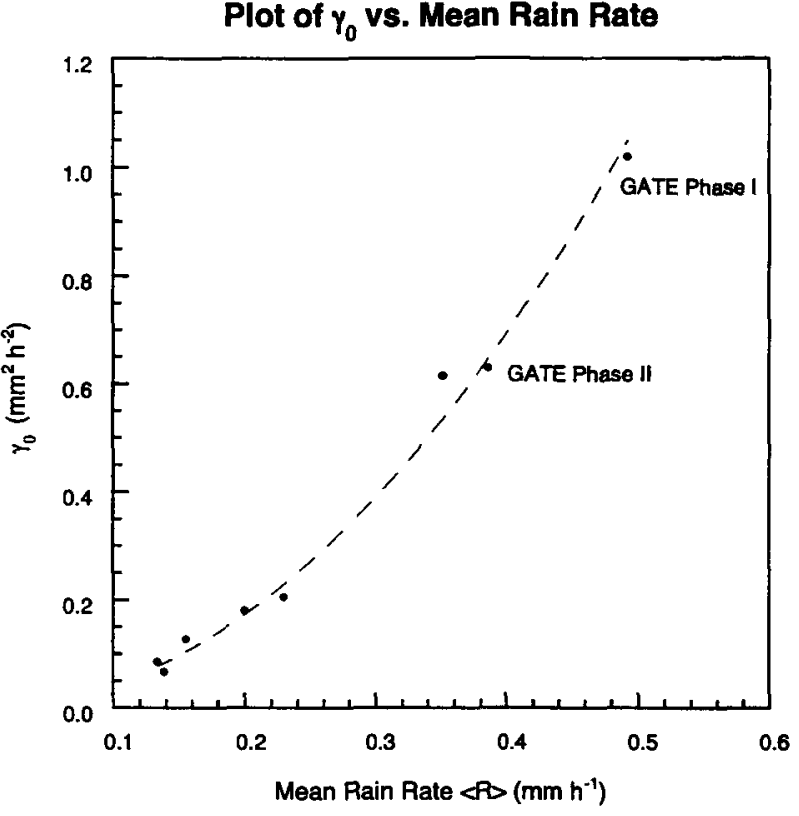

Figure 3. Plot of the parameter $\gamma_{0}$ against the mean rain rate $\langle R\rangle$ for the TOGA-COARE and GATE data sets. The continuous curve shows the least squares fit to the formula (38).

As discussed in section 2, an additional property of the conditional statistics of rain, namely the constancy of the dimensionless ratio $\mu$, allows one to draw some inferences about the asymptotic $L$-dependence of the function $p(L)$. We now turn to a discussion of this statistic for the TOGA-COARE data set. It is, however, worth pointing out that in doing so we are exploring territory that lies outside the scope of the original spectral model.

Our data set provides a simple way to test the constancy of the ratio $\mu$ defined by (34) as originally suggested by Short et al. [1993]. Eq. (35) implies that the plot of $1+\sigma^{2}(L) /\langle R\rangle^{2}$ vs. $1 / p(L)$ would be a straight line through the origin if $\mu$ is a constant independent of $L$. A scatterplot of all the data points from the six data sets (2 ships, 3 cruises) and each value of $L$ is shown in Figure 5. The linear fit seems satisfactory; the slope yields a mean value of $\mu \approx 3.03$ collectively for all the six data sets. However, there is a pronounced systematic deviation from the linear fit in the "large" $p(L)$ regime [i.e., $p(L) \simeq 1$ ], which corresponds to large $L$. One can also evaluate $\mu$ separately for each data set. The individual values were found to be within a narrow range, with a low value of 2.63 for MIT Cruise 2 and a high value of 3.39 for TOGA Cruise 2. As noted in 
Plot of integral correlation time vs. L for TOGA COARE
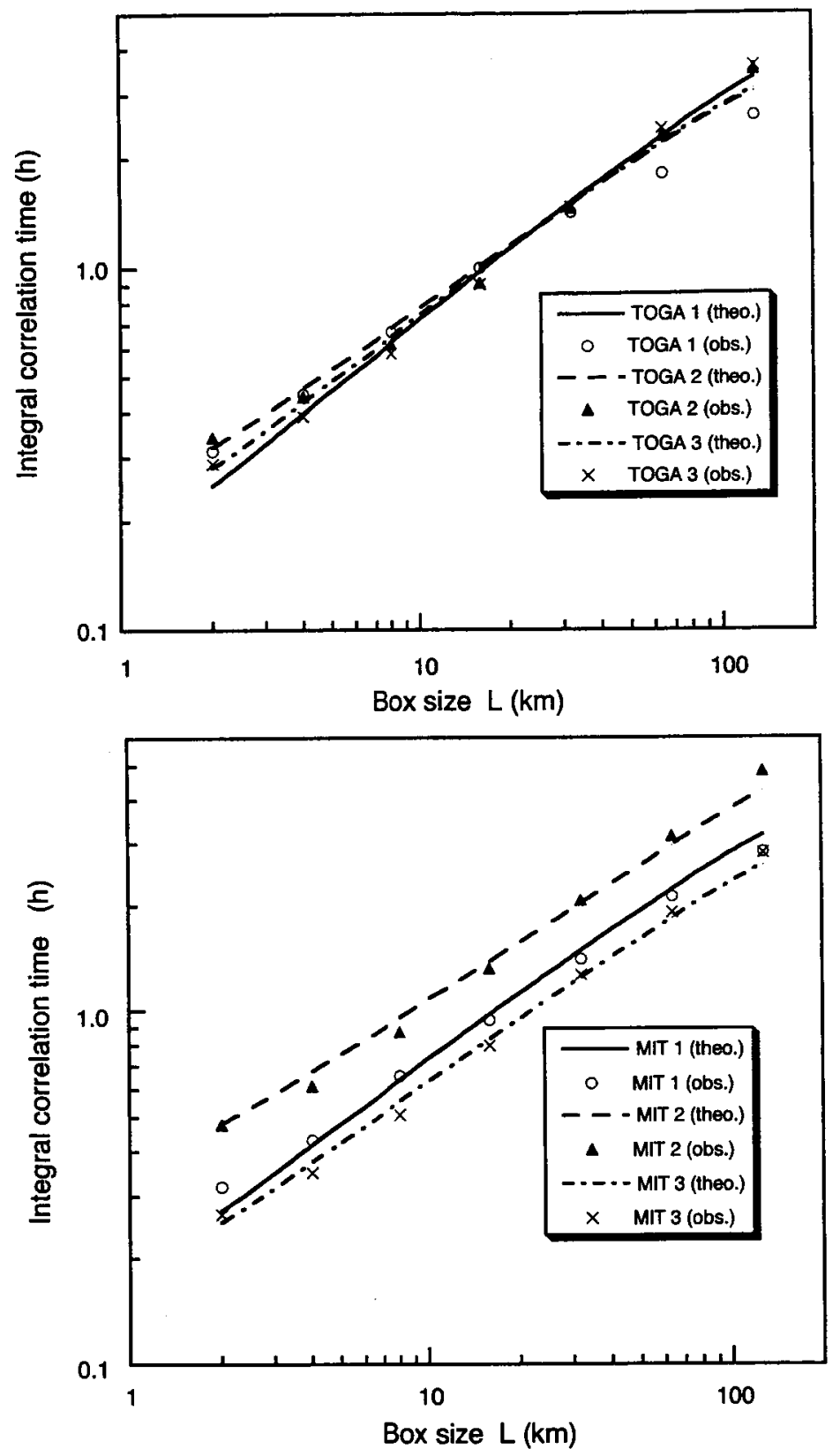

Figure 4. Plot of the integral correlation time $\hat{\tau}_{\text {int }}$ evaluated with a finite cut-off $\tau_{\max }$ for the three TOGA and MIT cruises and various box sizes $L$ ranging from $2 \mathrm{~km}$ to $128 \mathrm{~km}$. The curves represent interpolation of the values computed from the spectral model.

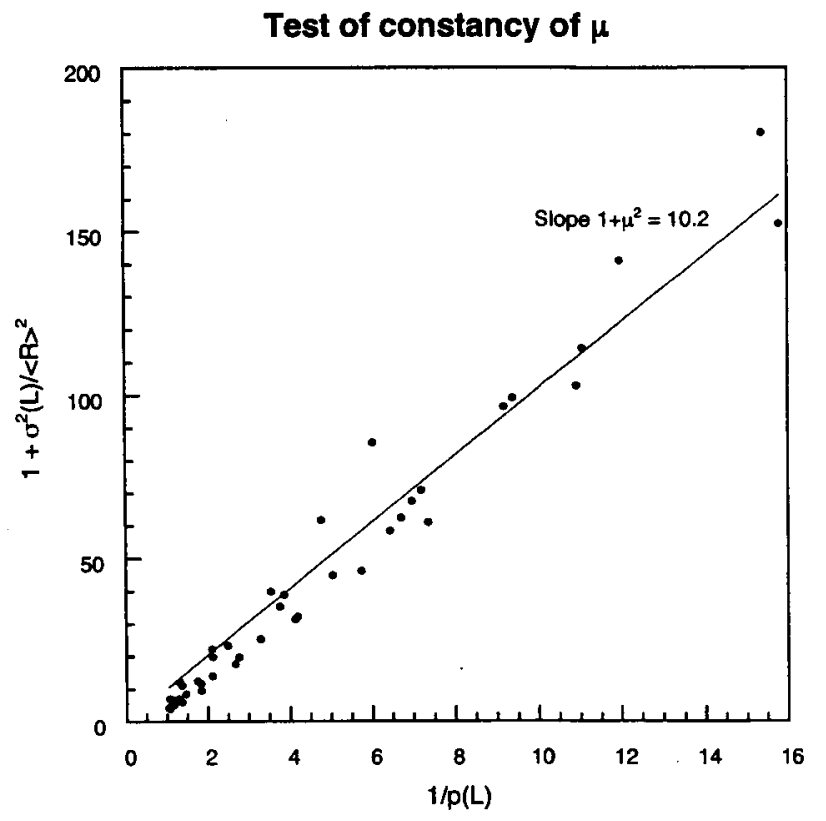

Figure 5. Scatterplot of $1+\sigma^{2}(L) /\langle R\rangle^{2}$ vs. $1 / p(L)$ for all the six TOGA-COARE data sets for averaging length scales $L$ ranging from $2 \mathrm{~km}$ to $128 \mathrm{~km}(6 \times 7=42$ data points). Least squares fit to a straight line through the origin yields the slope $1+\mu^{2} \approx 10.2$, i.e. $\mu \approx 3.03$ for the entire TOGA-COARE data set. 
section 2 , constancy of $\mu$ (or more generally, finiteness of $\mu$ as $L \rightarrow 0$ ) together with (22) leads to another important conclusion - a power law scaling behavior of the rain probability $p(L)$ at small $L$. Combining the formulas (22)-(24) and (38) with Eq. (35) we infer that for $L \ll L_{0}$,

$$
1 / p(L) \propto 1+\beta\left(L / 2 L_{0}\right)^{-2|\nu|},
$$

where

$$
\beta=\frac{2 \kappa \Gamma(|\nu|) H(\nu)}{1+\frac{1}{2} \kappa \Gamma(-|\nu|)},
$$

$H(\nu)$ is given by Eq. (21) and $\kappa$ is the dimensionless number introduced in (38). This immediately implies the power law behavior

$$
p(L) \sim\left(\frac{L}{L_{0}}\right)^{2|\nu|}
$$

as $L \rightarrow 0$, which can be independently checked from the data. Fits to a power law $p(L) \propto L^{\eta}$ for each of the six data sets are shown in Figure 6. A straight line fit on a plot of $\log p(L)$ vs. $\log L$ provides evidence of a power law behavior between $L=2 \mathrm{~km}$ and $64 \mathrm{~km}$. In Table 3 the observed exponent $\eta_{\text {obs }}$ is compared against the theoretically expected value $\eta_{\mathrm{th}}=2|\nu|$ for each data set. We see that, although there is generally speaking a close agreement between the two sets of values, for four of the six data sets $\eta_{\text {obs }}$ is somewhat larger than $\eta_{\text {th }}$ contrary to what one would infer from Eq. (35). However, it should be noted that according to the model the power law dependence on $L$ becomes accurate only in the scaling regime $L \ll L_{0}$. Consequently, it is more appropriate to compare $\eta_{\text {th }}$ with the power law exponent determined from $p(L)$ in the small $L$ limit. Indeed, a closer inspection of the graphs of $p(L)$ shows a slight but systematic deviation at small $L$ from the power law obtained by least squares fit, indicating a smaller slope there. In Table 3 we have also included an estimated exponent $\eta_{\mathrm{obs}}^{0}$ evaluated from the slope of a linear interpolation between $L=2 \mathrm{~km}$ and $8 \mathrm{~km}$, which clearly improves agreement between the model and observation. One can also see in retrospect that the constancy of $\mu$ which gave rise to the power law scaling behavior of $p(L)$, itself becomes progressively more accurate in the limit of small $L$.

For the GATE data we find that within the range of $L$ between $4 \mathrm{~km}$ and $56 \mathrm{~km}$ the rain probability $p(L)$ ( $L$ in $\mathrm{km}$ ) is represented quite well by the power law formulas

$$
p(L)=\left\{\begin{array}{ll}
0.122(L / 4)^{0.61} & \text { Phase I } \\
0.098(L / 4)^{0.68} & \text { Phase II }
\end{array},\right.
$$
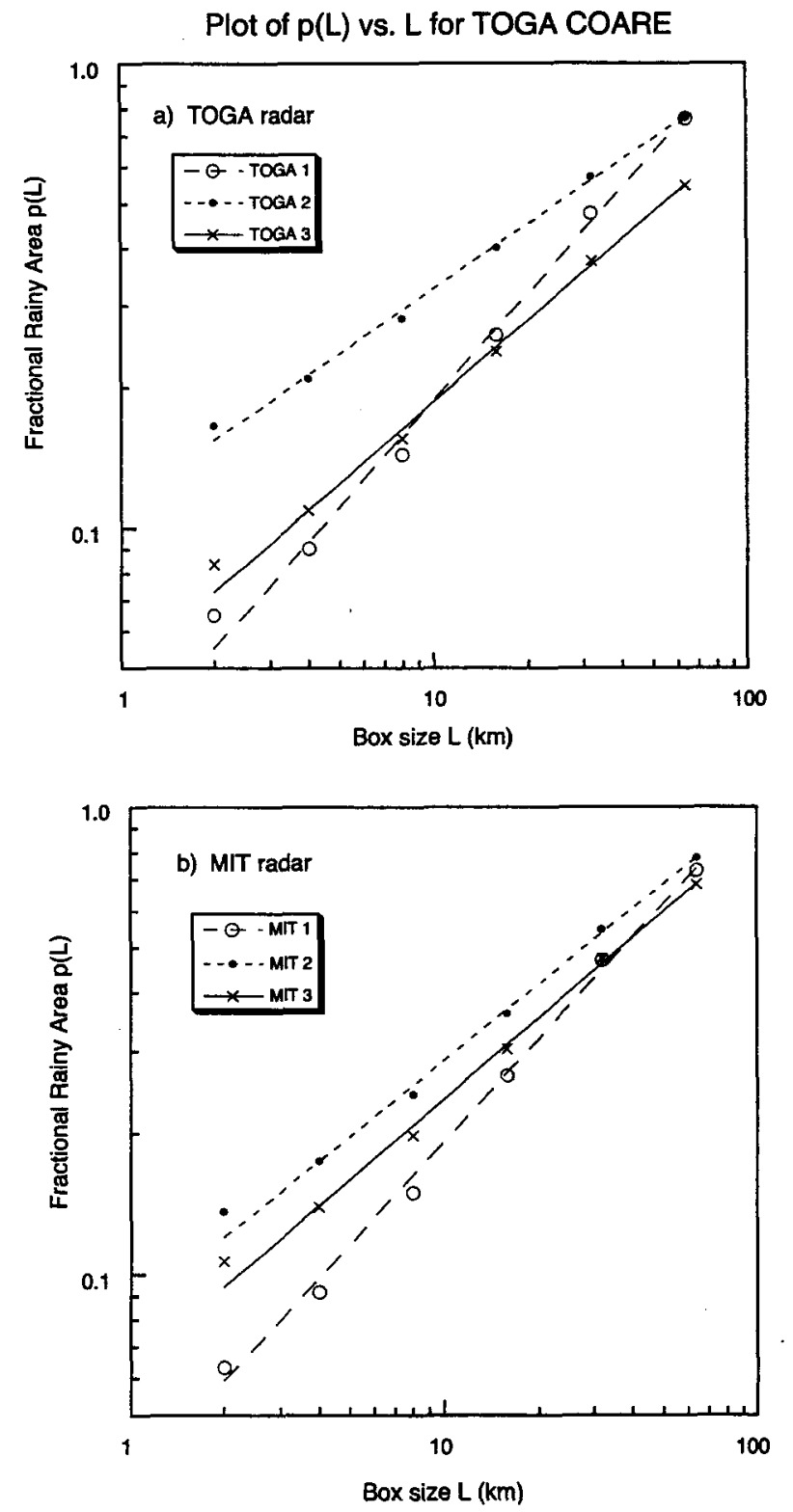

Figure 6. Power law fit to the fractional rainy area $p(L)$ for the three TOGA and MT cruises and various box sizes $L$ ranging from $2 \mathrm{~km}$ to $64 \mathrm{~km}$. The exponents are given in Table 3. 
Table 3. Comparison between theoretical and observed values of the exponent $\eta$.

\begin{tabular}{llll}
\hline \multicolumn{1}{c}{ Dataset } & $\eta_{\text {obs }}$ & $\eta_{\text {obs }}^{0}$ & $\eta_{\text {th }}=2|\nu|$ \\
\hline TOGA Cruise 1 & $0.760 \pm 0.026$ & 0.571 & $0.669 \pm 0.025$ \\
MIT Cruise 1 & $0.728 \pm 0.025$ & 0.618 & $0.595 \pm 0.047$ \\
TOGA Cruise 2 & $0.464 \pm 0.011$ & 0.382 & $0.477 \pm 0.057$ \\
MIT Cruise 2 & $0.540 \pm 0.014$ & 0.417 & $0.409 \pm 0.054$ \\
TOGA Cruise 3 & $0.582 \pm 0.015$ & 0.448 & $0.581 \pm 0.056$ \\
MIT Cruise 3 & $0.573 \pm 0.015$ & 0.448 & $0.519 \pm 0.063$ \\
\hline
\end{tabular}

Comparison between value of the power law index $\eta$ defined by the stipulated behavior $p(L) \propto L^{\eta}$ of the fractional rainy area $p(L)$ as function of the averaging length scale $L$ determined from the data and the value predicted by the model. The second column gives the "mean" exponent obtained by an overall least squares fit, while the third column gives the exponent determined from the slope at small $L$. In the last column specifying the "theoretical" value $\eta_{\mathrm{th}}=2|\nu|$ determined from the $\sigma^{2}(L)$ vs. $L$ plots [Figure 2 via the fit to (22)], we also include the error bars not reported in Table 1.

which are consistent with the expected behavior $p(L) \rightarrow$ 0 as $L \rightarrow 0$. [See also $H a$ et al., 2002, who use a linear regression fit to describe $p(L)$ as function of $L$ ]. The exponents are, however, in each case quite different from the values suggested by the spectral model fit. We also find that for GATE, the quantity $\mu(L)$ actually depends significantly on $L$. In fact, $\mu(L)$ changes from about 1.77 at $L=4 \mathrm{~km}$ to about 2.16 at $L=56 \mathrm{~km}$ for Phase I and from about 1.89 at $L=4 \mathrm{~km}$ to about 2.22 at $L=56 \mathrm{~km}$ for Phase II.

As in the TOGA-COARE case, the goodness of a simple power law fit is probably somewhat fortuitous for GATE as well, since deviations from a power law behavior are to be expected on the basis of the model because of the presence of "subdominant" terms in $1 / p(L)$ such as the constant term in (40) or other possible $L$ dependent terms arising from a weak $L$-dependence of $\mu(L)$. Upon attempting to fit the data for $p(L)$ to a form like (40) we find that the best fit values of the exponent $\eta$ are slightly smaller than the values given above. However, they still differ substantially from the model-inspired value $2|\nu|$. We have been unable to fully reconcile the apparent discrepancy between the two sets of exponents along the lines discussed above in connection with the TOGA-COARE data.

One particular aspect that we did not address in this paper is the dependence of $p(L)$ and other rain statistics on the value of the rain threshold, i.e., the smallest value of rain rate that is experimentally measurable. This is an important issue, since different instruments have different thresholds and hence threshold dependence can greatly complicate intercomparison efforts for rain rate averages, especially conditional averages.

\subsection{Discussion}

From Table 1 it is evident that the model parameters vary considerably among the data sets. These variations are almost certainly due in part to real secular variations in the rainfall pattern. As discussed by Short et al. [1997], the TOGA-COARE region of the tropical Western Pacific experiences planetary scale equatorial waves including an annual cycle, intraseasonal oscillations such as Madden-Julian oscillations [Lau and Chan, 1985; Madden, 1986; Nakazawa, 1995] and westerly wind bursts related to El Niño-Southern Oscillation (ENSO) events. The first half of Cruise 2 had an intensely active period associated with intraseasonal oscillation accompanied by passage of cloud superclusters and heavy rainfall. The rainfall time series during this period also shows a quasi 2-day oscillation of the type described by Takayabu [1994] and Takayabu et al. [1996]. They attribute this oscillation to westward-propagating inertio-gravity waves. By contrast, the spatial rainfall pattern during much of Cruise 1 appears to be much more homogeneous and isotropic, characteristic of small-scale convective systems. For a more detailed discussion see Short et al. [1997].

\section{Conclusion}

In this paper we have presented a model of second moment statistics of rainfall based on a linear stochas- 
tic dynamical equation. The model makes definite predictions about the statistical behavior of area-averaged rain rate in both space and time. In particular, the model predicts simple power law dependence of the rain statistics on the averaging area as the area tends to zero, perhaps reflecting a fractal nature of the rain field at smaller spatial scales. These predictions are tested with a radar-derived gridded tropical oceanic precipitation data set and the model is shown to describe the data quite well within the intrinsic limitations of the model assumption of statistical homogeneity, isotropy and stationarity. In particular, the intimate relationship between the dependence of the correlation length and time scales of the rain field on the spatial smoothing predicted by the model is in accordance with data. It should, however, be emphasized that in extrapolating the model-derived limiting behavior of the rain statistics deduced from a data set on a finite grid as the averaging scale goes to zero, we are effectively probing the "subgrid" structure of the rain field, i.e., the structure on scales finer than allowed by the instrumental resolution.

To conclude, we briefly mention some directions for future work. Like the spatially averaged rain rate, the spectral model also predicts relationships among the power law scaling exponents for the time-averaged rain rate as well. It would be interesting to see how well the model is capable of describing statistics of rain gauge data averaged over various time intervals. In a sequel to this paper, we will investigate the statistical properties of time-averaged local rain rates as measured by rain gauges using our model spectrum. Our model provides a convenient framework for addressing intercomparison problems that involve averaging the precipitation field over many different length and time scales. Recently the model has been used to investigate the problem of validation of satellite measurements of rainfall using rain gauge measurements on the ground [Bell and Kundu, 2003]. Finally, simple power law behavior of the various statistics of rain fields with respect to rescaling of the spatial averaging grid at small scales suggests a modelindependent explanation based entirely on the scaling properties of the various statistical quantities involved. We plan to explore this possibility in future publications.

\section{Appendix A: Analytic Formula for $\tau_{\text {int }}(L)$}

In this appendix we give a derivation of the formula (31) for the integral correlation time $\tau_{\text {int }}(L)$ and de- scribe its limiting behavior as $L \rightarrow 0$.

Equation (18) for the variance of the area-averaged rain rate in a grid box $\mathcal{A}$ of area $A=L^{2}$ reads

$$
\sigma_{A}^{2}=4 \gamma_{0} G\left(\nu ; L / L_{0}\right),
$$

where $G(\nu ; z)$ is defined as the double integral (19). On the other hand, the lagged autocovariance function $\mathcal{C}_{\mathcal{A A}}(0, \tau)$ is given by (BK96, Appendix A)

$$
\begin{aligned}
\mathcal{C}_{\mathcal{A} \mathcal{A}}(0, \tau)=\frac{2}{\pi} & \int_{0}^{\infty} \int_{0}^{\infty} d k_{1} d k_{2} \mathcal{G}^{2}\left(\frac{k_{1} L}{2}\right) \\
& \times \mathcal{G}^{2}\left(\frac{k_{2} L}{2}\right) c(\mathbf{k}, \tau)
\end{aligned}
$$

where $\mathcal{G}^{2}(x)=\sin ^{2} x / x^{2}$ is the Bartlett filter function and $c(\mathbf{k}, \tau)$ is give by Eq. (11). Setting $\tau=0$, we have an alternative integral representation of $\sigma_{A}^{2}$ :

$$
\begin{aligned}
\sigma_{A}^{2} & =\sqrt{\frac{2}{\pi}} F_{0} \int_{0}^{\infty} \int_{0}^{\infty} d k_{1} d k_{2} \mathcal{G}^{2}\left(\frac{k_{1} L}{2}\right) \mathcal{G}^{2}\left(\frac{k_{2} L}{2}\right) \tau_{k} \\
& =\sqrt{\frac{2}{\pi}} F_{0} \tau_{0} \int_{0}^{\infty} \int_{0}^{\infty} \frac{d k_{1} d k_{2} \mathcal{G}^{2}\left(k_{1} L / 2\right) \mathcal{G}^{2}\left(k_{2} L / 2\right)}{\left[1+\left(k_{1}^{2}+k_{2}^{2}\right) L_{0}^{2}\right]^{1+\nu}}
\end{aligned}
$$

where in the last step we have made use of the explicit form of $\tau_{k}$ given by (6). Comparing the two representations of $\sigma_{A}^{2}$ we obtain the useful integral identity

$$
\begin{aligned}
& \int_{0}^{\infty} \int_{0}^{\infty} \frac{d k_{1} d k_{2} \mathcal{G}^{2}\left(k_{1} L / 2\right) \mathcal{G}^{2}\left(k_{2} L / 2\right)}{\left[1+\left(k_{1}^{2}+k_{2}^{2}\right) L_{0}^{2}\right]^{1+\nu}} \\
= & \frac{2 \pi}{\Gamma(1+\nu) L_{0}^{2}} G\left(\nu ; L / L_{0}\right) .
\end{aligned}
$$

Next, consider the Fourier transform of $\mathcal{C}_{\mathcal{A A}}(0, \tau)$ :

$$
\begin{aligned}
\tilde{\mathcal{C}}_{A}(\omega)=\frac{2}{\pi} & \int_{0}^{\infty} \int_{0}^{\infty} d k_{1} d k_{2} \mathcal{G}^{2}\left(\frac{k_{1} L}{2}\right) \\
& \times \mathcal{G}^{2}\left(\frac{k_{2} L}{2}\right) \tilde{c}(k, \omega) .
\end{aligned}
$$

Using the explicit form of the model spectrum (7) at $\omega=0$, we get

$$
\begin{aligned}
& \tilde{\mathcal{C}}_{A}(\omega=0)=\frac{2}{\pi} F_{0} \int_{0}^{\infty} \int_{0}^{\infty} d k_{1} d k_{2} \mathcal{G}^{2}\left(\frac{k_{1} L}{2}\right) \\
& \times \mathcal{G}^{2}\left(\frac{k_{2} L}{2}\right) \tau_{k}^{2} \\
&=\frac{2}{\pi} F_{0} \tau_{0}^{2} \int_{0}^{\infty} \int_{0}^{\infty} \frac{d k_{1} d k_{2} \mathcal{G}^{2}\left(k_{1} L / 2\right) \mathcal{G}^{2}\left(k_{2} L / 2\right)}{\left[1+\left(k_{1}^{2}+k_{2}^{2}\right) L_{0}^{2}\right]^{2(1+\nu)}} .
\end{aligned}
$$


In view of the identity (A2) this reduces to

$$
\tilde{\mathcal{C}}_{A}(\omega=0)=4 \sqrt{\frac{2}{\pi}} \gamma_{0} \tau_{0} \frac{\Gamma(1+\nu)}{\Gamma(2+2 \nu)} G\left(1+2 \nu ; L / L_{0}\right),
$$

where we have also used (12) to substitute for $F_{0}$ in terms of the standard model parameters $\gamma_{0}, L_{0}$ and $\nu$. Using Eqs. (18), (A3) and (30) we obtain Eq. (31). The integrals were numerically evaluated with the help of Mathematica [v. 4; see Wolfram, 1999].

Finally, we examine the limiting behavior of $\tau_{\text {int }}(L)$ as $L \rightarrow 0$. We note that for the range of values of the exponent $\nu$ of interest to us, $-1 / 2<\nu<0$, (or $0<1+2 \nu<1$ ), Eq. (20) yields the asymptotic behavior

$$
G(\nu ; z)=\frac{1}{2} \Gamma(|\nu|) H(\nu)\left(\frac{z}{2}\right)^{-2|\nu|}+O(1)
$$

and

$$
G(1+2 \nu ; z)=\frac{1}{8} \Gamma(1+2 \nu)+O\left(z^{2(1+2 \nu)}\right),
$$

as $z \rightarrow 0$. From Eq. (31) it follows that $\tau_{\text {int }}(L)$ vanishes for $L \rightarrow 0$ as

$$
\tau_{\text {int }}(L) \sim \frac{\tau_{0}}{4} \frac{\Gamma(1+\nu)}{(1+2 \nu) \Gamma(|\nu|) H(\nu)}\left(\frac{L}{2 L_{0}}\right)^{2|\nu|} .
$$

In this limit the product $\tau_{\text {int }}(L) \sigma^{2}(L)$ approaches a constant :

$$
\lim _{L \rightarrow 0} \tau_{\text {int }}(L) \sigma^{2}(L) \simeq \frac{\gamma_{0} \tau_{0}}{2} \frac{\Gamma(1+\nu)}{1+2 \nu}
$$

\section{Appendix B: Effect of Finite Cut-off in the Evaluation of the Integral Correlation Time}

In this appendix we obtain an expression for the fractional error in the integral correlation time $\hat{\tau}_{\text {int }}$ predicted by the spectral model for a circular disk of radius $a$ as a function of the lag $\tau_{\max }$ at which numerical integration of the correlation function is stopped. The derivation is a simple extension of the calculations described in Bell and Kundu [2003].

The covariance of instantaneous area-averaged rain rate averaged over a circular disk of radius $a$ separated by a time interval $\tau$ is

$$
\begin{aligned}
\mathcal{C}_{\mathcal{A A}}(0, \tau)= & \frac{1}{A^{2}} \int_{A} d^{2} \mathbf{x} \int_{A} d^{2} \mathbf{y} c(\mathbf{x}-\mathbf{y}, \tau) \\
= & \frac{1}{A^{2}} \int_{A} d^{2} \mathbf{x} \int_{A} d^{2} \mathbf{y}(2 \pi)^{-1} \\
& \times \int d^{2} \mathbf{k} e^{i \mathbf{k} \cdot(\mathbf{x}-\mathbf{y})} c(\mathbf{k}, \tau) .
\end{aligned}
$$

The areal integrals can be done in a closed form. After some algebra using (9), one obtains

$$
\begin{gathered}
\mathcal{C}_{\mathcal{A A}}(0, \tau)=\frac{4 \gamma_{0} \Gamma(1+\nu)}{\alpha^{2}} \int_{0}^{\infty} \frac{d \kappa}{\kappa} \frac{J_{1}^{2}(\kappa \alpha)}{v(\kappa)} \\
\times e^{-\left|\tau / \tau_{0}\right| v(\kappa)} .
\end{gathered}
$$

where $\alpha=a / L_{0}$,

$$
v(\kappa)=\left(1+\kappa^{2}\right)^{1+\nu},
$$

and $J_{1}(x)$ is the Bessel function of order 1 . For a circular disk of area $L^{2}$ the radius is $a=L / \sqrt{\pi}$.

Using (B1) one can easily show that

$$
\begin{aligned}
\tau_{\text {int }} & =\frac{\int_{0}^{\infty} d \tau \mathcal{C}_{\mathcal{A} \cdot \mathcal{A}}(0, \tau)}{\sigma_{A}^{2}} \\
& =\tau_{0}\left[\frac{\int_{0}^{\infty} \frac{d \kappa}{\kappa} \frac{J_{1}^{2}(\kappa \alpha)}{v^{2}(\kappa)}}{\int_{0}^{\infty} \frac{d \kappa}{\kappa} \frac{J_{1}^{2}(\kappa \alpha)}{v(\kappa)}}\right] .
\end{aligned}
$$

If we estimate $\tau_{\text {int }}$ by introducing a finite upper cut-off in the $\tau$-integral, then the fractional error $\epsilon\left(\tau_{0} ; \tau_{\max }\right)$ defined as

$$
\epsilon\left(\tau_{0} ; \tau_{\max }\right)=\frac{\tau_{\text {int }}-\hat{\tau}_{\text {int }}}{\tau_{\text {int }}} .
$$

is given by the formula

$$
\epsilon\left(\tau_{0} ; \tau_{\max }\right)=\frac{\int_{0}^{\infty} \frac{d \kappa}{\kappa} \frac{J_{1}^{2}(\kappa \alpha)}{v^{2}(\kappa)} \exp \left[-\left(\tau_{\max } / \tau_{0}\right) v(\kappa)\right]}{\int_{0}^{\infty} \frac{d \kappa}{\kappa} \frac{J_{1}^{2}(\kappa \alpha)}{v^{2}(\kappa)}} .
$$

Eq. (B5) is numerically evaluated using Mathematica [Wolfram, 1999] to estimate the correction to the integral correlation time discussed in section 3.3. A plot of $\epsilon$ against the ratio $\tau_{\max } / \tau_{0}$ is shown in Figure $\mathrm{B} 1$ for typical parameter values.

\section{Appendix C: Estimate of Random Error for the Integral Correlation Time of an AR(1) Process}

In this appendix we obtain a simple estimate of the random error of the integral correlation time of a normally distributed random variable undergoing a stationary, linear, first order autoregressive [AR(1)] process, evaluated from a single time series of finite length $T$.

Consider the time series $\{x(t)\}$ of length $T$ generated by a single realization of a random variable $X(t)$ undergoing a linear stationary $\operatorname{AR}(1)$ process for which the lagged covariance function

$$
\gamma(\tau)=\left\langle X^{\prime}(t+\tau) X^{\prime}(t)\right\rangle
$$




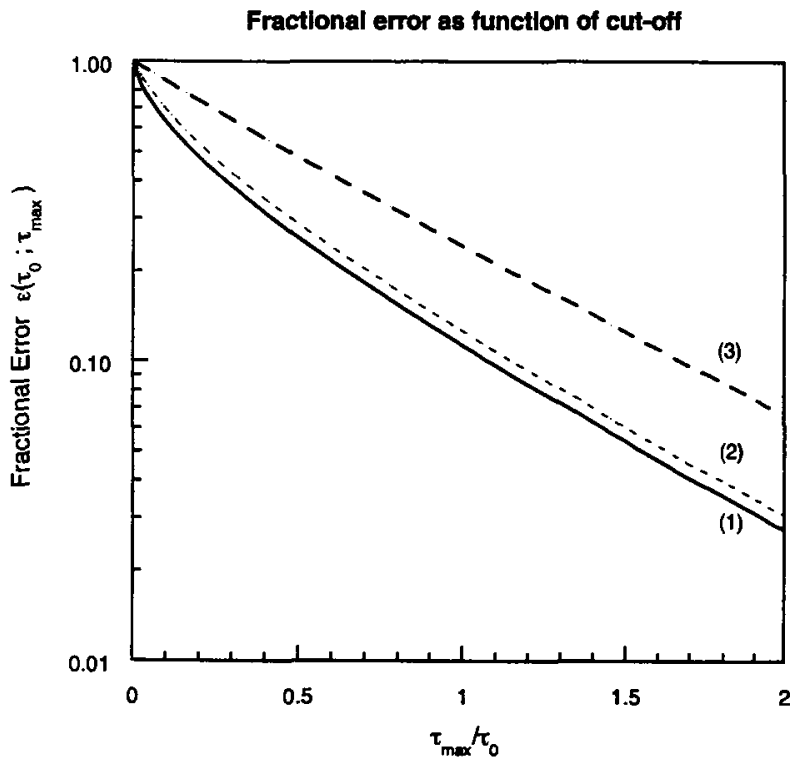

Figure B1. Plot of the fractional error (B5) as function of the ratio $\tau_{\max } / \tau_{0}$ computed for the typical spectral model parameters $\nu=-0.25$ and $L_{0}=70 \mathrm{~km}$ and three values of disk radius $a$. The curves marked (1), (2) and (3) represent the results for the radii $a=1 \mathrm{~km}, 10 \mathrm{~km}$ and $100 \mathrm{~km}$. The corresponding true integral correlation times are $0.052 \tau_{0}, 0.19 \tau_{0}$ and $0.65 \tau_{0}$ respectively. has the simple exponential form

$$
\gamma(\tau)=\sigma^{2} e^{-|\tau| / \tau_{\Lambda}}
$$

Its estimate, obtained from a finite time series, namely,

$$
c(\tau)=\frac{1}{T} \int_{0}^{T} d t x^{\prime}(t+\tau) x^{\prime}(t)
$$

can be thought of as a random variable

$$
Y(\tau)=\frac{1}{T} \int_{0}^{T} d t X^{\prime}(t+\tau) X^{\prime}(t)
$$

depending on the realization of $X(t)$. We are interested in estimating the random error for the integral correlation time represented by the random variable

$$
T_{\mathrm{int}}=\int_{0}^{\tau_{\max }} Z(\tau) d \tau
$$

where the variable $Z(\tau)=Y(\tau) / Y(0)$ represents the lagged correlation function. The desired error estimate is simply the square root of the variance

$$
\sigma^{2}\left[T_{\text {int }}\right]=\left\langle T_{\text {int }}^{2}\right\rangle-\left\langle T_{\text {int }}\right\rangle^{2},
$$

which can then be expressed in the form

$$
\sigma^{2}\left[T_{\mathrm{int}}\right]=\int_{0}^{\tau_{\max }} \int_{0}^{\tau_{\max }} d \tau_{1} d \tau_{2}\left\langle Z^{\prime}\left(\tau_{1}\right) Z^{\prime}\left(\tau_{2}\right)\right\rangle .
$$

For simplicity of notation we write

$$
Y(\tau)=A, Y(0)=B .
$$

These random variables can each be expressed as the sum of respective mean values and fluctuations:

$$
A=a+\alpha, B=b+\beta,
$$

where

$$
a \equiv\langle A\rangle=\gamma(\tau), b \equiv\langle B\rangle=\sigma^{2} .
$$

Up to second order in the fluctuations one finds

$$
\left\langle Z^{\prime}\left(\tau_{1}\right) Z^{\prime}\left(\tau_{2}\right)\right\rangle \approx \frac{1}{b^{2}}\left\langle\alpha_{1} \alpha_{2}+\frac{\beta^{2}}{b^{2}} a_{1} a_{2}\right\rangle,
$$

where the subscripts 1 and 2 indicate evaluation at lags $\tau_{1}$ and $\tau_{2}$ respectively.

Eq. (C6) can be recast in a much simpler form if $X(t)$ (and therefore $X^{\prime}(t)$ ) is a gaussian random variable. Using the relation [see, e.g., Anderson, 1958]

$$
\begin{aligned}
& \left\langle X^{\prime}\left(t_{1}\right) X^{\prime}\left(t_{2}\right) X^{\prime}\left(t_{3}\right) X^{\prime}\left(t_{4}\right)\right\rangle= \\
& \quad\left\langle X^{\prime}\left(t_{1}\right) X^{\prime}\left(t_{2}\right)\right\rangle\left\langle X^{\prime}\left(t_{3}\right) X^{\prime}\left(t_{4}\right)\right\rangle \\
& +\quad\left\langle X^{\prime}\left(t_{1}\right) X^{\prime}\left(t_{3}\right)\right\rangle\left\langle X^{\prime}\left(t_{2}\right) X^{\prime}\left(t_{4}\right)\right\rangle \\
& +\left\langle X^{\prime}\left(t_{1}\right) X^{\prime}\left(t_{4}\right)\right\rangle\left\langle X^{\prime}\left(t_{2}\right) X^{\prime}\left(t_{3}\right)\right\rangle,
\end{aligned}
$$


which expresses the fourth moment of such a variable in terms of products of the second moment, the quantity

$$
\left\langle\alpha_{1} \alpha_{2}\right\rangle=\left\langle Y^{\prime}\left(\tau_{1}\right) Y^{\prime}\left(\tau_{2}\right)\right\rangle
$$

can be reduced to the form

$$
\begin{gathered}
\left\langle\alpha_{1} \alpha_{2}\right\rangle=\frac{1}{T^{2}} \int_{0}^{T} \int_{0}^{T} d t_{1} d t_{2}\left[\gamma\left(t_{1}-t_{2}+\tau_{1}-\tau_{2}\right) \gamma\left(t_{1}-t_{2}\right)\right. \\
\left.+\gamma\left(t_{1}-t_{2}+\tau_{1}\right) \gamma\left(t_{1}-t_{2}-\tau_{2}\right)\right] .
\end{gathered}
$$

The double integrals can be simplified with the help of the integral identity

$$
\int_{0}^{T} \int_{0}^{T} d t_{1} d t_{2} f\left(t_{1}-t_{2}\right)=\int_{-T}^{T} d \tau(T-|\tau|) f(\tau),
$$

which, for a function $f(\tau)$ decaying exponentially as $\tau \rightarrow \infty$, in the approximation $T \gg \tau_{A}$ further simplifies to

$$
\int_{0}^{T} \int_{0}^{T} d t_{1} d t_{2} f\left(t_{1}-t_{2}\right) \approx T \int_{-\infty}^{\infty} d \tau^{\prime} f\left(\tau^{\prime}\right)
$$

Making use of the explicit form of the lagged covariance function $\gamma(\tau)$ given by Eq. (C2) and carrying out the remaining integration, we get

$$
\begin{aligned}
\left\langle\alpha_{1} \alpha_{2}\right\rangle \approx & \frac{\sigma^{4}}{T}\left[\left(\left|\tau_{1}-\tau_{2}\right|+\tau_{A}\right) e^{-\left|\tau_{1}-\tau_{2}\right| / \tau_{A}}\right. \\
& \left.+\left(\tau_{1}+\tau_{2}+\tau_{A}\right) e^{-\left(\tau_{1}+\tau_{2}\right) / \tau_{A}}\right]
\end{aligned}
$$

Setting $\tau_{1}=\tau_{2}=0$, we also get

$$
\left\langle\beta^{2}\right\rangle \approx \frac{2 \sigma^{4} \tau_{A}}{T} .
$$

With Eqs. (C6), (C10) and (C11), Eq. (C5) can be drastically simplified using the identity (C9) again with $\tau_{\max }$ instead of $T$. In the approximation $\tau_{\max } \gg \tau_{0}$, we finally obtain the desired formula

$$
\sigma^{2}\left[T_{\text {int }}\right] \approx \frac{4 \tau_{\max } \tau_{A}^{2}}{T}\left[1+O\left(\tau_{A} / \tau_{\max }\right)\right],
$$

which is Eq. (39), since $\tau_{\text {int }}=\tau_{A}$ for the exponentially decaying correlation $(\mathrm{C} 2)$.

Acknowledgments. We thank the anonymous reviewers for their constructive comments which greatly improved the paper. This research was supported by the Office of Earth Science of the National Aeronautics and Space Administration as part of the Tropical Rainfall Measuring Mission.

\section{References}

Anderson, T. W., An Introduction to Multivariate Statistical Analysis, John Wiley \& Sons, 1958.

Bell, T. L., Climate sensitivity from Fluctuation Dissipation: Some simple model tests, J. Atmos. Sci., 37, 1700-1707, 1980.

Bell, T. L., A space-time stochastic model of rainfall for satellite remote-sensing studies, J. Geophys. Res., 92D, 9631-9643, 1987.

Bell, T. L., A. Abdullah, R. L. Martin and G. R. North, Sampling errors for satellite-derived tropical rainfall: Monte Carlo study using a space-time stochastic model, J. Geophys. Res., 95D, 2195-2205, 1990.

Bell, T. L., and N. Reid, Detecting the diurnal cycle of rainfall using satellite observations, J. Appl. Meteor., 32, 311-322, 1993.

Bell, T. L., and P. K. Kundu, A study of the sampling error in satellite rainfall estimates using optimal averaging of data and a stochastic model, J. Climate, 9, 1251-1268, 1996.

Bell, T. L., P. K. Kundu and C. D. Kummerow, Sampling errors of SSM/I and TRMM rainfall averages: Comparison with error estimates from surface data and a simple model, J. Appl. Meteor., 40, 938-954, 2001.

Bell, T. L., and P. K. Kundu, Comparing satellite rainfall estimates with rain-gauge data: Optimal strategies suggested by a spectral model, J. Geophys. Res., 108(D3), 4121, doi:10.1029/2002JD002641, 2003.

Benson, D. A., S. W. Wheatcraft, and M. M. Meerschaert, The fractional- order governing equation of Lévy motion, Water Resour. Res., 36, 1413-1423, 2000.

Cox, D. R., and V. Isham, A simple spatial-temporal model of rainfall, Proc. R. Soc. Lond., A 415, 317-328, 1988.

Deidda, R., Rainfall downscaling in a space-time multifractal framework, Water Resour. Res., 36, 1779-1794, 2000.

Foufoula-Georgiou, E. and and W. F. Krajewski, Recent advances in rainfall modeling, estimation and forecasting, Rev. Geophys., 33, 1125-1137, 1995.

Gupta, V. K., and E. C. Waymire, A stochastic kinematic study of subsynoptic space-time rainfall, Water Resour. Res., 15, 637-644, 1979.

Gupta, V. K., and E. C. Waymire, Multiscaling properties of spatial rainfall and river flow distributions, J. Geophys. Res., 95D, 1999-2009, 1990.

Ha, E., G. R. North, C. Yoo and K.-J. Ha, Evaluation of some ground truth designs for satellite estimates of rain rate, J. Atmos. Oceanic Tech., 19, 65-73, 2002.

Jenkins, G. M., and D. G. Watts, Spectral Analysis and its applications, Holden-Day, San Francisco, 1968.

Kedem, B., L. S. Chiu and G. R. North, Estimation of mean rain rate: Application to satellite observations, J. Geophys. Res., 95D, 1965-1972, 1990.

Lau, K.-M. and P. H. Chan, Aspects of the 40-50-day oscillation in the tropics, Mon. Wea. Rev., 113, 1889-1909, 1985. 
Laughlin, C. R., On the effect of temporal sampling on the observation of mean rainfall, in Precipitation Measurements from Space, Workshop Report, edited by D. Atlas and O. W. Thiele, pp. D59-D66, available from Goddard Space Flight Center, Greenbelt, MD 20771, 1981.

Le Cam, L., A stochastic description of precipitation, in IV Berkeley Symposium in Mathematical Statistics and Probability, vol. 3, pp. 165-186, University of California, Berkeley, 1961.

Lovejoy, S. Area-perimeter relations for rain and cloud areas, Science, 216, 185-187, 1982.

Lovejoy, S. and B. Mandelbrot, Fractal properties of rain and a fractal model, Tellus, 37A, 209-232, 1985.

Madden, R. A., Seasonal variations of the 40-50-day oscillation in the tropics, J. Atmos. Sci., 43, 3138-3158, 1986.

Marsan, D., D. Schertzer, and S. Lovejoy, Causal space-time multifractal processes: predictability and forecasting of rain fields, $J$. Geophys. Res., 101D, 26,333-26,346, 1996.

Marshak, A., A. Davis, R. Cahalan, and W. Wiscombe, Bounded cascade models as nonstationary fractals, Phys. Rev. A, 49, 55-69, 1994.

McConnell, A. , and G. R. North, Sampling errors in satellite estimates of tropical rain, J. Geophys. Res., 92D, 95679570, 1987.

Menabde, M., D. Harris, A. W. Seed, G. Austin and D. Stow, Multiscaling properties of rainfall and bounded random cascades, Water Resour. Res., 33, 2823-2830, 1997.

Nakazawa, T., Intraseasonal oscillations during the TOGA COARE IOP, J. Meteor. Soc. Japan, 73, 305-319, 1995.

North, G. R., and S. Nakamoto, Formalism for comparing rain estimation designs, J. Atmos. Oceanic Tech., 6, 985992, 1989.

Over, T. M., and V. K. Gupta, A space-time theory of mesoscale rainfall using random cascades, $J$. Geophys. Res., 101D, 26,319-26,331, 1996.

Schertzer, D., and S. Lovejoy, Physical modeling and analysis of rain and clouds by anisotropic scaling of multiplicative processes, J. Geophys. Res., 92D, 9693-9714, 1987.

Schertzer, D., S. Lovejoy, F. Schmitt, Y. Chigirinskaya and D. Marsan Multifractal cascade dynamics and turbulent intermittency, Fractals, 5(3), 421-471, 1997.

Seed, A. W., R. Srikanthan and M. Menabde, A space-time model for design storm rainfall, J. Geophys. Res., 104D, 31,623-31,630, 1999.

Short, D. A., D. B. Wolff, D. Rosenfeld, and D. Atlas, A study of the threshold method utilizing raingage data, $J$. Appl. Meteorol., 32, 1379-1387, 1993.

Short, D. A., P. A. Kucera, B. S. Ferrier, J. A. Gerlach, S. A. Rutledge, and O. W. Thiele, Shipborne radar rainfall patterns within the TOGA COARE IFA, Bull. Amer. Meteorol. Soc., 78, 2817-2836, 1997.

Smith, J. A., and W. F. Krajewski, Statistical modeling of space-time rainfall using radar and rain gage observations, Water Resour. Res., 23, 1893-1900, 1987.

Takayabu, Y. N., Large-scale cloud disturbances associated with equatorial waves. Part II: Westward propagating inertio-gravity waves, J. Meteor. Soc. Japan, 72, 451465, 1994.

Takayabu, Y. N., K.-M. Lau, and C.-H. Sui, Two-day variation in cloud-atmosphere coupled system observed in TOGA COARE IOP, Mon. Wea. Rev., 124, 1892-1913, 1996.

Venugopal, V., E. Foufoula-Georgiou, and V. Sapozhnikov, Evidence of dynamic scaling in space-time rainfall, $J$. Geophys. Res., 104D, 31,599-31,610, 1999.

Waymire, E., V. K. Gupta, and I. Rodríguez-Iturbe, A spectral theory of rainfall intensity at the meso- $\beta$ scale, Water Resour. Res., 20, 1453-1465, 1984.

Waymire, E., Scaling limits and self-similarity in precipitation fields, Water Resour. Res., 21, 1271-1281, 1985.

Webster, P. J., and R. Lukas, TOGA COARE: The Coupled Ocean-Atmosphere Experiment, Bull. Amer. Meteorol. Soc., 73, 1377-1416, 1992.

Wheater, H. S., V. S. Isham, D. R. Cox, R. E. Chandler, A. Kakou, P. J. Northrop, L. Oh, C. Onof, and I. Rodriguez-Iturbe, Spatial-temporal rainfall fields: modelling and statistical aspects, Hydrol. Earth System Sci., 4, 581-601, 2000.

Wolfram, S., The Mathematica Book, 4th ed., Wolfram Media/Cambridge University Press, 1999.

Yoo, C., J. B. Valdés, and G. R. North, Stochastic modeling of multidimensional precipitation fields considering spectral structure, Water Resour. Res., 32, 2175-2187, 1996.

Prasun K. Kundu and Thomas L. Bell, NASA / Goddard Space Flight Center, Mail Code 913, Greenbelt, MD 20771, U.S.A. (kundu@climate.gsfc.nasa.gov; bell@climate.gsfc.nasa.gov)

This preprint was prepared with AGU's IATEX macros v5.01, with the extension package 'AGU'++' by $P$. W. Daly, version $1.6 \mathrm{~b}$ from 1999/08/19. 